Research Paper

\title{
RIP3 blockade prevents immune-mediated hepatitis through a myeloid-derived suppressor cell dependent mechanism
}

\author{
Man Liu1,2\#, Hongxia Zhang1\#, Lu Zhang1\#, Xin Liu1, Simin Zhou1, Xiaoyi Wang1, Weilong Zhong1, Jie \\ Zhang $^{1}$, Bangmao Wang ${ }^{1 凶}$, Jingwen Zhao ${ }^{1 凶}$ and Lu Zhou ${ }^{1,3}$ \\ 1. Department of Gastroenterology and Hepatology, General Hospital, Tianjin Medical University, Tianjin, China. \\ 2. Department of Physiology and Pathophysiology, Tianjin Medical University, Tianjin, China. \\ 3. Department of Gastroenterology and Hepatology, People's Hospital of Hetian District, Xinjiang Uygur Autonomous Region, China. \\ \#These authors have contributed equally to this work. \\ $\bowtie$ Corresponding authors: E-mails: lzhou01@tmu.edu.cn (L.Z.); jingwenzhao@tmu.edu.cn (J.Z.); gi.tmuh@sohu.com (B.W).
}

( ) The author(s). This is an open access article distributed under the terms of the Creative Commons Attribution License (https://creativecommons.org/licenses/by/4.0/). See http://ivyspring.com/terms for full terms and conditions.

Received: 2021.07.27; Accepted: 2021.10.16; Published: 2022.01.01

\begin{abstract}
Autoimmune hepatitis $(\mathrm{AlH})$ is an immune-mediated chronic inflammatory liver disease, and its pathogenesis is not fully understood. Our previous study discovered that receptor interacting protein kinase 3 (RIP3) is correlated with serum transaminase levels in AlH patients. However, its role and underlying mechanism in AlH are poorly understood. Here, we detected the increased expression and activation of RIP3 in livers of patients and animal models with AIH. The inhibition of RIP3 kinase by GSK872 prevented concanavalin A (ConA)-induced immune-mediated hepatitis (IMH) by reduced hepatic proinflammatory cytokines and immune cells including Th17 cells and macrophages. Further experiments revealed that RIP3 inhibition resulted in an increase in $\mathrm{CDI} / \mathrm{b}^{+} \mathrm{Grl}{ }^{+}$myeloid-derived suppressor cells (MDSCs) with immunoregulatory properties in the liver, spleen, and peripheral blood. Moreover, the depletion of $\mathrm{Gr}-1+$ MDSCs abrogated the protective effect and immune suppression function of GSK872 in ConA-induced IMH. Altogether, our data demonstrate that RIP3 blockade prevents ConA-induced IMH through promoting MDSCs infiltration. Inhibition of RIP3 kinase may be a novel therapeutic avenue for AlH treatment.
\end{abstract}

Key words: immune-mediated hepatitis, receptor-interacting protein kinase 3, myeloid-derived suppressor cells, cytokines and chemokines, glucocorticoid treatment

\section{Introduction}

Autoimmune hepatitis (AIH) is a progressive immune-mediated liver disorder characterized by elevated serum transaminases, interface hepatitis, the presence of circulating autoantibodies, and hypergammaglobulinemia [1-4]. Originally AIH was believed to be a disease of young women. Currently it is known that $\mathrm{AIH}$ can present at all ages, moreover, the rate of cirrhosis at diagnosis of $\mathrm{AIH}$ was similar at all ages [5]. The etiology of AIH is incompletely understood, and clinical treatments rely on chronic administration of high doses of prednisolone (occasionally associated with an immunosuppressant such as azathioprine). However, these agents, particularly corticosteroids, can be associated with important side effects, such as metabolic syndrome with its sequelae and osteoporosis [6-8]. Thus, further understanding of the disease's cellular and molecular mechanisms may assist in identifying better treatment modalities, aid the limitation of adverse effects from therapy, and improve prognostication.

Immune response imbalance is one of the major hallmarks of AIH. Excessive activation of macrophages and imbalance between $\mathrm{T}$ regulatory cells (Tregs) and $\mathrm{T}$ effector cells contribute to the perpetuation of liver damage in AIH [9-12]. Concanavalin A (ConA)-induced hepatitis is an 
appropriate animal model for immune-mediated liver injury in human $\mathrm{AIH}$ [13-15]. A growing evidence supports the critical role of inflammatory cells, including T cells, natural killer (NK) cells, NK T-cells, and monocytes/macrophages, in the pathophysiology of AIH in animal and human research [16, 17]. Thus, if the function of these cells can be regulated and inhibited, then liver inflammation can be attenuated.

Myeloid-derived suppressor cells (MDSCs) have emerged as major regulators of inflammation and immune function in a variety of pathological conditions [18-20]. MDSCs can repress the activation of $\mathrm{T}$ cells, B cells, macrophages, and NK cells but activate Tregs [21-23]. Recent findings suggest that dampening MDSC suppressor function is an attractive strategy for optimizing cancer immunotherapy and contributing to enhancing the sensitivity of anti-tumor immunotherapy [19, 24, 25]. Conversely, enhancing or sustaining MDSCs suppressive function would be beneficial in inhibiting inflammation and immune disorders [26]. Accumulating evidence indicates that MDSCs can protect against liver injury in $\mathrm{AIH}$ patients and experimental immune-mediated hepatitis (IMH) [27-29]. Therefore, the regulation of MDSCs is a promising direction to ameliorate immune hepatitis. However, the methods to successfully maintain MDSCs in the desired suppressive state in $\mathrm{AIH}$ remain poorly defined.

Receptor interacting protein kinase 3 (RIP3) is a molecular switch between tumor necrosis factor (TNF)-induced apoptosis and necrosis [30]. We previously showed that RIP3 signaling is highly correlated with serum transaminase levels in patients with $\mathrm{AIH}$, indicating that RIP3 signal plays important roles in the pathogenesis of the disease [31]. On the other hand, RIP3 also targets and delivers negative signals to MDSCs recruitment in tumor research [32, 33]. However, its role in AIH remains largely unknown. Therefore, in this present study, we investigated the role of RIP3 in IMH. Activation of RIP3 were detected readily in livers of patients and animal models with AIH. RIP3 blockade protected mice against IMH through a mechanism mediated by $\mathrm{CD}_{11} \mathrm{~b}^{+} \mathrm{Gr}-\mathrm{1}^{+}$MDSCs. In addition, the expression of RIP3 in livers of IMH mice was significantly decreased by glucocorticoid treatment, suggesting that RIP3 is a potential target for the treatment of AIH.

\section{Materials and methods}

\section{Participants}

A total of eight $\mathrm{AIH}$ patients and five controls with hepatic cyst were recruited from the Department of Gastroenterology and Hepatology in Tianjin Medical University General Hospital between January 2019 and August 2019. The enrolled AIH patients fulfilled the 1999 revised International Autoimmune Hepatitis Group (IAIHG) classification criteria of AIH [34]. No patients were treated with corticosteroids. Sex and age-matched controls fulfilled the following inclusion criteria: (1) cyst diameter $<4 \mathrm{~cm}$ and cyst fluid is clear, (2) normal ranges of liver function test, (3) normal histopathology of liver tissue next to the cyst, (4) an absence of hepatitis B/C virus antigen. Liver biopsy specimens was obtained from all participants. This study was approved by the Ethics Committee of Tianjin Medical University General Hospital, and all participants provided signed informed consents.

\section{Animal experiments}

Female C57BL/6 mice (6-week-old) were obtained from the Institute of Laboratory Animal Science, Chinese Academy of Medical Sciences \& Peking Union Medical College and maintained under specific pathogen-free environment. All the mice were acclimatized for 1 week and then randomized into four groups ( $\mathrm{n}=6-7$ per group). According to the previous studies $[35,36]$, mice were intravenously (i.v.) injected with ConA (15 mg/kg; Solarbio, Beijing, China) for $12 \mathrm{~h}$ to establish a mouse model of IMH. To evaluate whether RIP3 blockade prevents IMH, the mice were i.p. injected with GSK872 $(1 \mathrm{mg} / \mathrm{kg}$; Selleck, USA), dexamethasone (DEX, $1 \mathrm{mg} / \mathrm{kg}$; Sigma, USA), or an equal volume of vehicle (phosphatebuffered saline (PBS) containing 2\% dimethyl sulfoxide) $1 \mathrm{~h}$ before intravenous injection of ConA (15 mg/kg; Solarbio, Beijing, China). Control mice were given PBS alone. Blood samples were collected $12 \mathrm{~h}$ after ConA administration by retro-orbital bleeding, and the mice were sacrificed. Livers and spleens were harvested.

For MDSCs depletion, mice were i.p. injected with a depleting anti-mouse Gr-1 antibody (250 $\mu \mathrm{g} /$ mouse; BioXCell, USA) or an isotype control (250 $\mu \mathrm{g} /$ mouse; BioXCell, USA) $36 \mathrm{~h}$ before ConA administration [37, 38]. GSK872 were i.p. given $1 \mathrm{~h}$ before ConA treatment. All animal studies were performed in accordance with the National Institutes of Health Guide for the Care and Use of Laboratory Animals. The animal use protocol has been reviewed and approved by the Animal Ethical and Welfare Committee of Tianjin Medical University, Approval No. IRB2020-WZ-175.

\section{Assay for serum aminotransferase activity}

Alanine aminotransferase (ALT) and aspartate aminotransferase (AST) activities were measured by 
standard procedures at the Institute of Clinical Chemistry of the Tianjin Medical University General hospital.

\section{Analysis of liver index and spleen index}

Liver index was calculated as (liver weight (g)/body weight (g)) ${ }^{*} 100 \%$. Spleen index was calculated as (spleen weight $(\mathrm{g}) /$ body weight $(\mathrm{g})$ ) * $100 \%$.

\section{Real-time quantitative polymerase chain reaction (RT-qPCR)}

Total RNA was extracted from liver using TRIzol reagent (Invitrogen, USA). cDNA was synthesized using TIANScript RT Kit (Tiangen, Beijing, China) following the manufacturer's protocol. qPCR analysis was performed using SYBR Green PCR Master Mix (ThermoScientific) and specific primers (Genewiz, Beijing, China). Table 1 shows the oligonucleotide primers for target genes. All values were normalized to the levels of Glyceraldehyde-3-phosphate dehydrogenase (GAPDH). The relative mRNA expression of the target gene was calculated by standard $\Delta \Delta \mathrm{Ct}$ method.

Table 1. The primer sequences used in RT-qPCR

\begin{tabular}{lll}
\hline Gene & Forward Sequence & Reverse Sequence \\
\hline RIP3 & GAAGACACGGCACTCCTTGGT & CTTGAGGCAGTAGTTCTTGGTG \\
& A & G \\
MLKL & CTGAGGGAACTGCTGGATAGA & CGAGGAAACTGGAGCTGCTGA \\
& G & T \\
TNFa & GAAGTTCCCAAATGGCCTCC & GTGAGGGTCTGGGCCATAGA \\
IL-6 & ACAACCACGGCCTTCCCTACTT & CACGATTTCCCAGAGAACATG \\
& & TG \\
IL-1 3 & CTTTGAAGTTGACGGACCC & TGAGTGATACTGCCTGCCTG \\
NLRP3 & TCACAACTCGCCCAAGGAGGA & AAGAGACCACGGCAGAAGCTA \\
& A & G \\
IFNy & CGGCACAGTCATTGAAAGCCT & GTTGCTGATGGCCTGATTGTC \\
& A & \\
iNOS & TCTAGTGAAGCAAAGCCCAAC & TGGCCTTGTGGTGAAGAGTG \\
CCL2 & AGCTGTAGTTTTGTCACCAAG & GTGCTGAAGACCTTAGGGCA \\
& C & \\
CCL3 & CCCAGCCAGGTGTCATTTC & GTGGCTACTTGGCAGCAAAC \\
CCL5 & TGCTGCTTTGCCTACCTCTC & TCCTTCGAGTGACAAACACGA \\
CCL7 & CGCTGCTTTCAGCATCCAAG & CTTGAAGATAACAGCTTCCCA \\
& & GG \\
CCL17 & CAATGTAGGCCGAGAGTGCTG & GCATCCCTGGAACACTCCACTG \\
CXCL1 & CTGCACCCAAACCGAAGTC & AGCTTCAGGGTCAAGGCAAG \\
GAPDH & GGCAAATCAACGGCACAGT & CGCTCCTGGAAGATGGTGAT \\
\hline
\end{tabular}

\section{Immunofluorescence}

Liver tissues were cut, deparaffinized, dehydrated, and incubated with specific antibodies against p-RIP3 (Abcam, USA), p-MLKL (Abcam, USA) and CD68 (Abcam, USA) overnight at $4{ }^{\circ} \mathrm{C}$. Subsequently, the sections were washed thrice with 1 $\times$ PBS and then incubated with fluorochromeconjugated secondary antibody for $60 \mathrm{~min}$ at room temperature in the dark. 4, 6-Diamidino-2phenylindole was finally applied to dye the nucleus. A fluorescence microscope DM5000 B (Leika,
Germany) was used for analysis.

\section{Histopathology}

The formalin-fixed liver tissues were routinely dehydrated, equilibrated, embedded in paraffin, and then cut into sections. Paraffin sections $(2 \mu \mathrm{m})$ were stained with H\&E and examined under light microscopy to determine the level of inflammation.

\section{Western blot analysis}

The liver tissues were lysed using RIPA lysis buffer containing protease inhibitors and phosphatase inhibitor (Solarbio, Beijing, China). Proteins were separated using sodium dodecyl sulphatepolyacrylamide gel electrophoresis system and transferred onto polyvinylidene difluoride membranes. The membranes were incubated overnight at $4{ }^{\circ} \mathrm{C}$ with specific primary antibodies: anti-RIP3 Ab (Santa Cruz), anti-MLKL Ab (CST, USA), anti-phospho-RIP3 Ab (CST, USA), antiphospho-MLKL Ab (CST, USA), and anti-GAPDH Ab (CST, USA). After washing in Tris-buffered saline and Tween 20, the membranes were incubated with horseradish peroxidase-conjugated secondary antibodies (CST, USA). Visualization was achieved using an ECL plus detection system (Solarbio, Beijing, China). ImageJ software was used for quantified analysis.

\section{Mononuclear cell isolation for flow cytometry}

HMNCs were isolated by the method of Diao et al. [27]. with minor modifications. Briefly, mouse livers were minced and digested with collagenase IV (Solarbio, Beijing, China) for $45 \mathrm{~min}$ at $37^{\circ} \mathrm{C}$. The cell suspensions were filtered with $70 \mu \mathrm{m}$ nylon cell strainers (Solarbio, Beijing, China) and centrifuged at $300 \mathrm{~g}$ for $5 \mathrm{~min}$. The pellet was washed with PBS and resuspended in $40 \%$ Percoll. The cell suspension was gently overlaid onto 70\% Percoll and centrifuged for 20 min at 750 g. Purified HMNCs were collected from the interphase and washed twice in PBS. Spleens and peripheral blood from mice were collected, grinded, and filtered. After depleting erythrocytes, purified mononuclear cells were collected for further flow cytometry analysis.

\section{Flow Cytometric Analysis}

The mononuclear cells were stained with zombie NIR $^{\mathrm{TM}}$ (BioLegend, USA) to distinguish live cells from dead cells, and incubated with Fc block (BioLegend, USA) for $10 \mathrm{~min}$ at room temperature. Then cells were washed, and incubated with the following fluorescently conjugated mAbs (BD Bioscience, USA): fluorescein isothiocyanate (FITC) anti-CD45, PEcy7 anti-CD11b, AF®647 anti-F4/80, PE anti-Gr-1, BV421 anti-CXCR2, for $45 \mathrm{~min}$ at $4{ }^{\circ} \mathrm{C}$ in the dark. In all 
experiments, appropriate isotype control IgGs were used. FACSverse flow cytometer (BD Bioscience, USA) and FlowJo software were used for analysis.

For CD4 ${ }^{+} \mathrm{IL}-17^{+} \mathrm{T}$ cells, cells were stimulated by cell activation cocktail (BioLegend, USA) in incubator $\left(5 \% \mathrm{CO}_{2}, 37^{\circ} \mathrm{C}\right)$ for $6 \mathrm{~h}$. Subsequently, using zombie NIR ${ }^{\mathrm{TM}}$ to distinguish live cells from dead cells, and incubated with Fc block. Cells were incubated with FITC-anti-CD4 (BD Bioscience, USA), and then cells were labeled with PE-anti-IL-17A (BD Bioscience, USA) after fixation and permeabilization.

For $\mathrm{CD}^{+}{ }^{+} \mathrm{CD} 25^{+}$Foxp $^{+}$cells, cells were stained with zombie NIR ${ }^{\mathrm{TM}}$ to distinguish live cells from dead cells and then incubated with Fc block. Subsequently, cells were labeled with FITC-anti-CD4 (BD Bioscience, USA) and PE/Cyanine7-anti-CD25 (BD Bioscience, USA). Then cells were labeled with Alexa Fluor647Foxp3 (BD Bioscience, USA) after fixation and permeabilization.

\section{T cell proliferation assay}

Hepatic CD11b ${ }^{+} \mathrm{Gr}-1^{+}$MDSCs and splenic $\mathrm{CD} 4^{+} \mathrm{T}$ cells from different groups were purified by Cell Isolation Kit II (Miltenyi Biotec, Germany) with $>90 \%$ purity according to the manufacturer's instruction. CD4 ${ }^{+}$Tcells were labeled with $2 \mu \mathrm{M}$ CFSE (Invitrogen, USA). Diverse MDSCs were co-cultured with CFSE-labeled $\mathrm{CD} 4^{+} \mathrm{T}$ cells at 1:2 ratio stimulated with anti-CD3/CD28 activating antibodies (Miltenyi Biotec, Germany). And $\mathrm{T}$ cell proliferation was determined after $72 \mathrm{~h}$ of coculture by flow cytometry.

\section{Statistical analysis}

Data are expressed as mean \pm standard deviation (SD). The data for two-group comparisons were assessed using two-tailed Student's $t$ test (parametric data). Differences among more than two groups were tested by one-way ANOVA. $P<0.05$ was considered statistically significant. Statistical analysis was performed on SPSS 22.0.

\section{Results}

\section{Activation of RIP3 in liver tissues of AIH patients and IMH mice}

Initially, we analyzed the expression of p-RIP3 and p-mixed lineage kinase domain-like protein (MLKL), which are the phosphorylated active forms of RIP3 and its downstream target MLKL, respectively, in paraffin-embedded liver sections of patients with $\mathrm{AIH}$ and controls by immunofluorescence. As shown in Figure 1A-B, p-RIP3positive cells and p-MLKL-positive cells markedly increased in the liver sections from $\mathrm{AIH}$ patients, particularly in CD68-positive macrophages, but staining was virtually absent in the control livers, thus indicating that RIP3 signaling is activated during AIH.

Livers from ConA-treated mice were analyzed for evidence of RIP3 activation to corroborate the above results. As shown in Figure 1C-E, Western blotting analysis of liver extracts showed that the expressions of $\mathrm{p}$-RIP3 and p-MLKL were significantly higher in mice treated with ConA in comparison with the controls, further proving that IMH associates with the activation of RIP3 signaling.

\section{RIP3 blockade protects mice against immune- mediated liver injury}

Next, we examined whether blockading RIP3 can prevent IMH. We took advantage of GSK872, a highly potent and selective RIP3 kinase inhibitor. Mice were pretreated intraperitoneally (i.p.) with GSK872, DEX, or vehicle $1 \mathrm{~h}$ before ConA administration and were sacrificed $12 \mathrm{~h}$ later. As shown in Figure 2A-C, induction of IMH by ConA was followed by elevated p-RIP3 and p-MLKL activities in the livers. Pretreatment of mice with GSK872 significantly reduced the expressions of p-RIP3 and p-MLKL, indicating that RIP3 activation can be effectively inhibited by RIP3 inhibitor. Notably, the activation of $p-R I P 3$ and $p-M L K L$ induced by ConA administration can be inhibited by DEX treatment, suggesting that RIP3 signaling is targeted by immunosuppressant DEX. Likewise, the overexpression of RIP3 and MLKL mRNA induced by ConA administration were abolished by GSK872 or DEX pretreatment (Figure 2D).

The serum levels of ALT and AST increased in ConA-injected mice were significantly reduced by GSK872 or DEX pretreatment (Figure 2E). Although the liver index was not significantly altered, the spleen index and size increased in ConA-injected mice, and the results can be reversed by GSK872 or DEX pretreatment (Figure 2F-H). Moreover, hematoxylin and eosin (H\&E) staining of liver sections showed reduced infiltration of inflammatory cells and minimal necrosis in mice injected with ConA and receiving GSK872 or DEX (Figure 2H). Collectively, these data show the effectiveness of inhibiting RIP3 in preventing IMH, consistent with the therapeutic effects of DEX.

\section{RIP3 blockade reduces hepatic levels of pro- inflammatory cytokines and immune cells during IMH}

To investigate the mechanism by which RIP3 blockade prevents immune-induced liver injury, we examined the proinflammatory cytokines and immune cells involved in IMH. As shown in Figure $\mathbf{3 A}$, the induction of liver damage by ConA was 
accompanied by significant upregulation of $\mathrm{TNFa}$, interleukin (IL)-6, IL-1 $\beta$, interferon (IFN) $\gamma$, and NLR family pyrin domain containing 3 (NLRP3), which can be attenuated by pretreatment with GSK872. Next, we determined whether RIP3 blockade changes immune cell infiltration in the livers. Flow cytometry analysis revealed that ConA inducement increased the accumulation of $\mathrm{CD}^{+} \mathrm{IL}-17^{+}$Th17 cells, $\mathrm{CD} 4^{+} \mathrm{CD} 25^{+}$ Foxp3 $3^{+}$Tregs and $\mathrm{CD}_{4} 5^{+} \mathrm{F} 4 / 80^{+}$macrophages (Figure
3B-D). Pretreatment with GSK872 was associated with decreased CD4 ${ }^{+} \mathrm{IL}_{-1} 7^{+}$Th17 cells and $\mathrm{CD} 45^{+} \mathrm{F} 4 / 80^{+}$macrophages and increased infiltration of $\mathrm{CD}^{+}{ }^{+} \mathrm{CD} 25^{+} \mathrm{Foxp}^{+}$Tregs. Altogether, these data support that RIP3 blockade inhibits the production of inflammatory cytokines and infiltration of proinflammatory immune cells but promotes the accumulation of $\mathrm{CD}^{+} \mathrm{CD}_{2} 5^{+}$Foxp3 $^{+}$Tregs with immunoregulatory properties in livers of IMH mice.

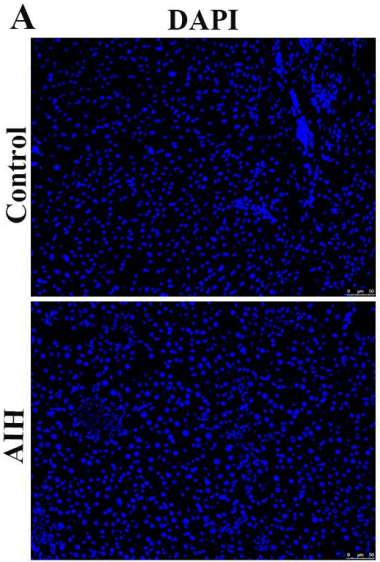

B
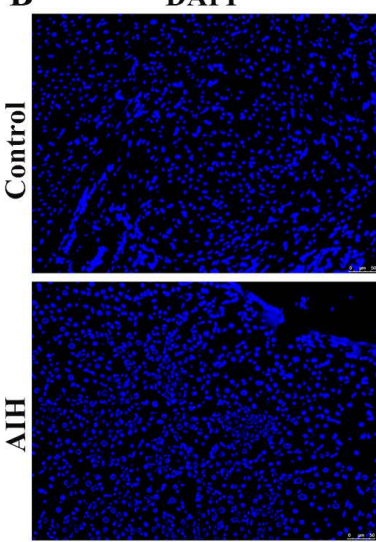

C

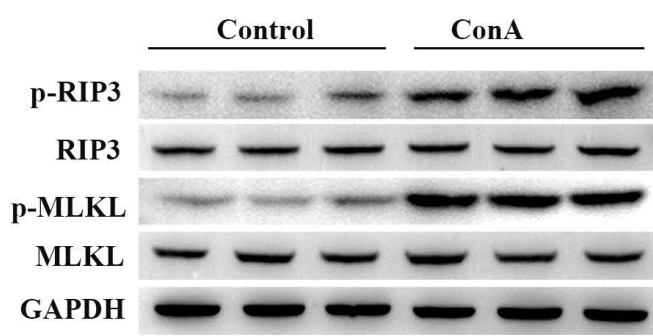

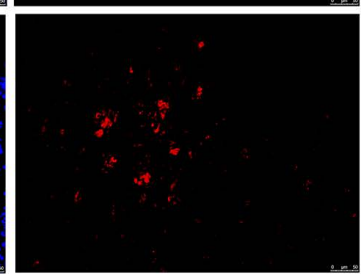

CD68
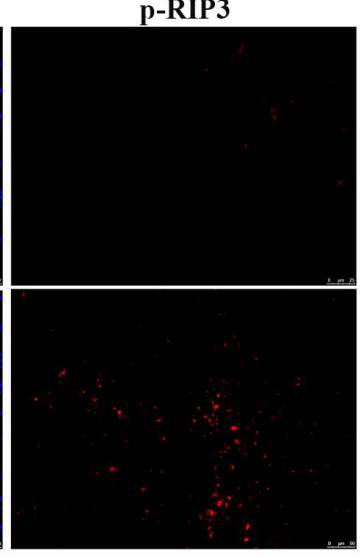

p-MLKL
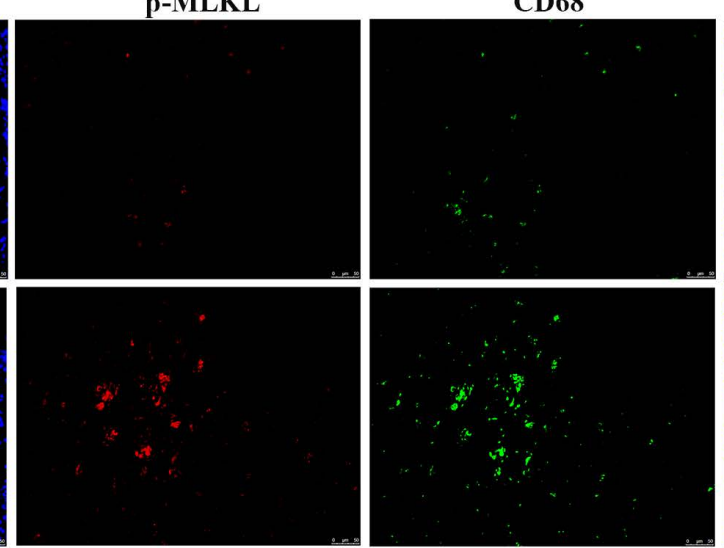

D

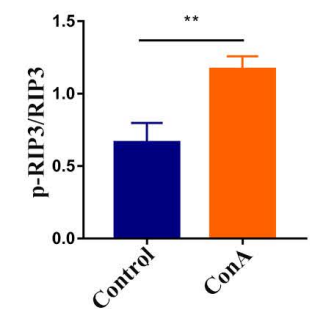

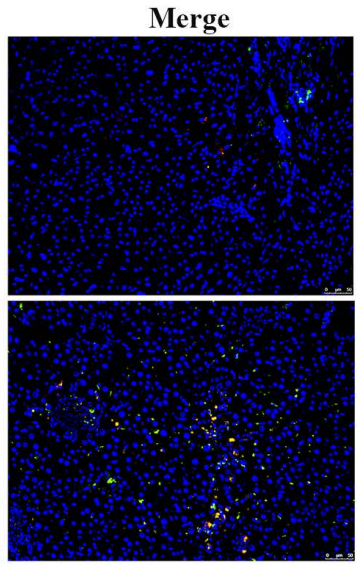

Merge
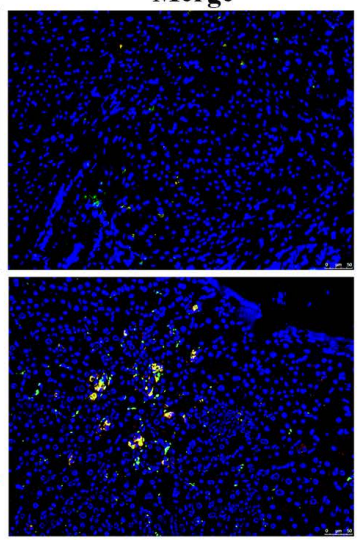

$\mathbf{E}$

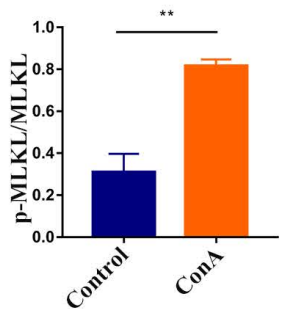

Figure 1. Activation of RIP3 signaling in the livers of AIH patients and IMH mice. Representative P-RIP3 (A) and p-MLKL (B) stained liver biopsy sections from control (hepatic cyst, $n=5$ ) and AlH patients $(n=8)$. Original magnification, $\times 200$; scale bars, $50 \mu \mathrm{m}$. (C) Mice, which were injected intravenously (i.v.) with ConA or vehicle $(n=7$ per group), were sacrificed at the indicated time points, and livers were collected and prepared for extraction of total proteins. Representative Western blots showing the expression levels of p-RIP3, RIP3, p-MLKL, and MLKL. GAPDH was used as loading control. Quantitative increases in p-RIP3 (D) and p-MLKL (E). All the values are shown as mean $\pm S D ; n s$, not significant; $* P<0.05 ; * * P<0.01$. 
A

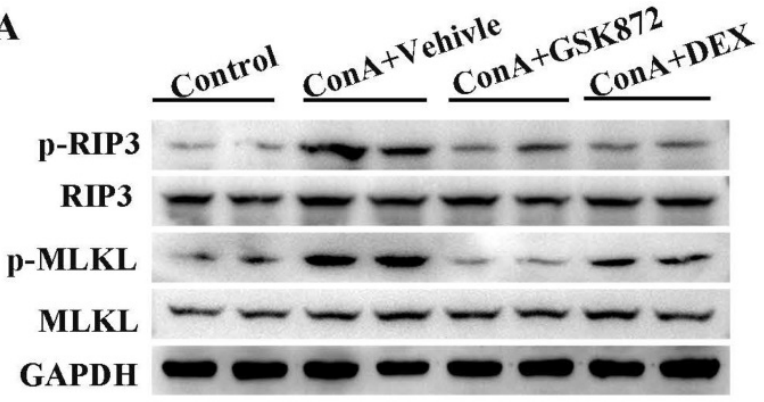

D

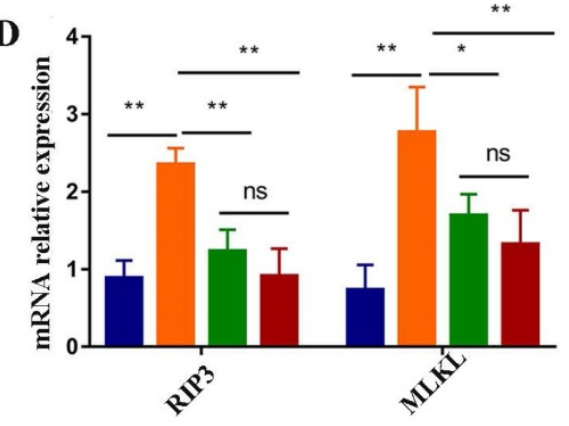

$\mathbf{E}$

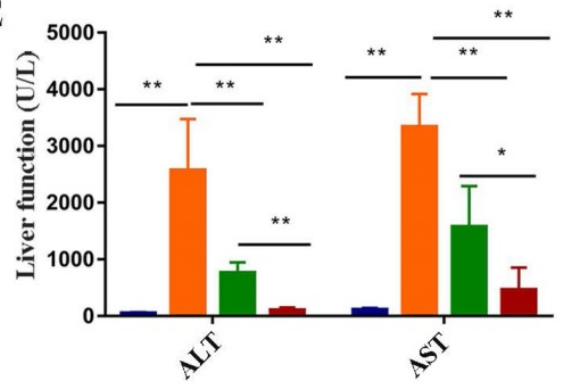

H
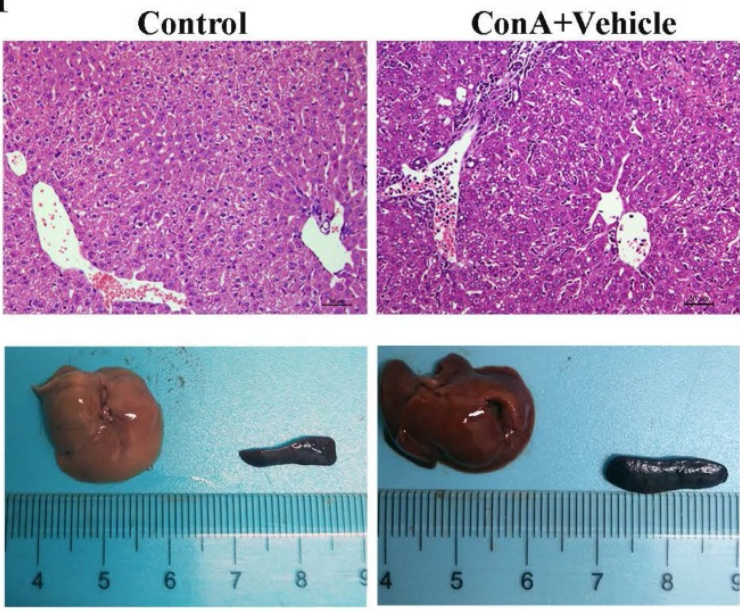

B
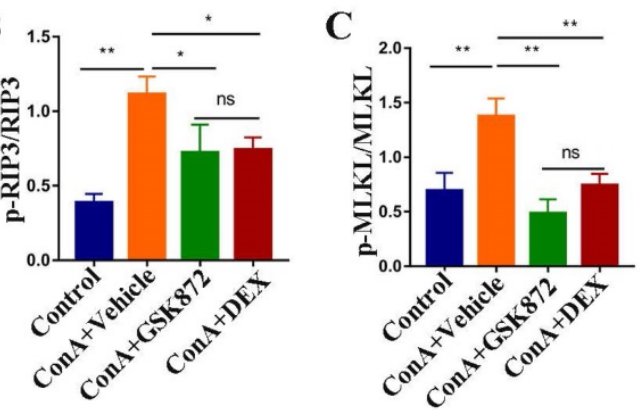

- Control

- ConA+Vehicle

- ConA+GSK872

- ConA+DEX

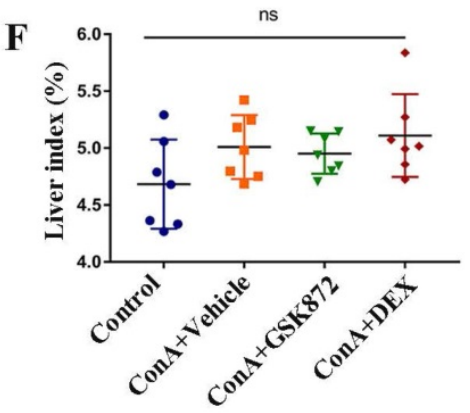

G

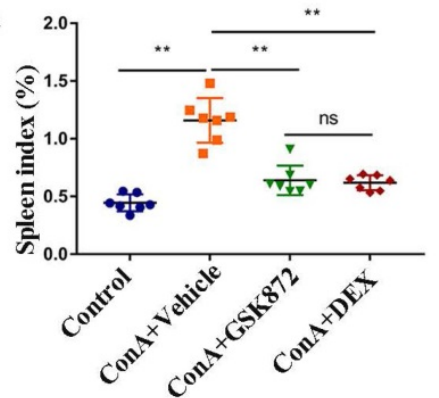

- ConA+Vehicle

- ConA+GSK872

- ConA+DEX

Figure 2. RIP3 blockade protects mice against immune-mediated liver injury. Mice were injected i.p. with GSK872 or DEX or vehicle $1 \mathrm{~h}$ before treatment with ConA. Control mice were given PBS alone ( $n=7$ per group). (A) Immunoblot analyses of $p-R I P 3$, RIP3, $p$-MLKL, and MLKL protein levels in the livers. GAPDH served as loading control. Quantitative expressions of p-RIP3 (B) and P-MLKL (C). (D) RIP3 and MLKL mRNA levels were assessed by RT-qPCR. Gene expression was normalized to GAPDH levels. (E) Serum levels of ALT and AST were measured $12 \mathrm{~h}$ after ConA treatment. The liver index $(\mathbf{F})$ and spleen index $(\mathbf{G})$ were determined $12 \mathrm{~h}$ after ConA treatment. (H) Representative photomicrographs (H\&E staining; original magnification 200x; scale bars, $50 \mu \mathrm{m}$; up) and perusal images (down) of livers in four groups. All the values are shown as mean \pm SD. ns, not significant; $* P<0.05, * * P<0.01$.

\section{RIP3 blockade promotes accumulation of CD 11 b+Gr-1+ MDSCs in the liver, spleen, and peripheral blood of IMH mice}

$\mathrm{CD}_{11} \mathrm{~b}^{+} \mathrm{Gr}-1^{+}$cells, termed as MDSCs, are a heterogeneous subset of myeloid cells with a potent immune-suppressive ability [39]. To evaluate whether RIP3 blockade induces the accumulation of MDSCs to prevent ConA-induced hepatitis, we employed flow cytometry to detect the frequency of $\mathrm{CD} 11 \mathrm{~b}^{+} \mathrm{Gr}-1^{+}$ MDSCs in mice livers, spleens, and peripheral blood. Compared with the controls, the frequency of 
CD $11 b^{+} G r-1^{+}$MDSCs increased in livers and spleens but reduced in the peripheral blood of ConA-injected mice (Figure 4A-D). The upregulation of frequency of CD11 $b^{+}$Gr- $1^{+}$MDSCs in livers and spleens induced by ConA was further increased by pre-treatment with GSK872. Moreover, the populations of $\mathrm{CD} 11 \mathrm{~b}^{+} \mathrm{Gr}-1^{+}$ MDSCs in peripheral blood of ConA-injected mice were strongly upregulated by GSK872 pretreatment. Another RIP3 inhibition GSK843 confirmed the effect of RIP3 blockade on $\mathrm{CD}_{11} \mathrm{~b}^{+} \mathrm{Gr} 1^{+}$MDSCs accumulation in ConA treated-mice (Figure S1). Altogether, these data demonstrate that RIP3 blockade promotes MDSCs accumulation in ConA-induced hepatitis.

Next, to examine whether they have the immunosuppressive properties, we isolated hepatic
CD11 $b^{+} G r-1^{+}$MDSCs from each group of mice and cocultured them with splenic $\mathrm{CD}^{+}{ }^{+} \mathrm{T}$ cells isolated from wild mice in the presence of anti-CD3 and anti-CD28 antibodies. The results showed that $\mathrm{CD} 11 \mathrm{~b}^{+} \mathrm{Gr}-1^{+}$cells isolated from each group strongly suppressed the proliferation of $\mathrm{CD} 4^{+} \mathrm{T}$ cells compared with when $\mathrm{T}$ cells alone were activated, which confirmed their immunosuppressive capacities (Figure 4E-F). However, there was no difference in the ability of hepatic MDSCs from different groups to inhibit $\mathrm{T}$ cell proliferation, demonstrating that RIP3 inhibition does not directly influence MDSCs suppressive function but rather increases the immunosuppressive environment via MDSCs expansion during IMH.
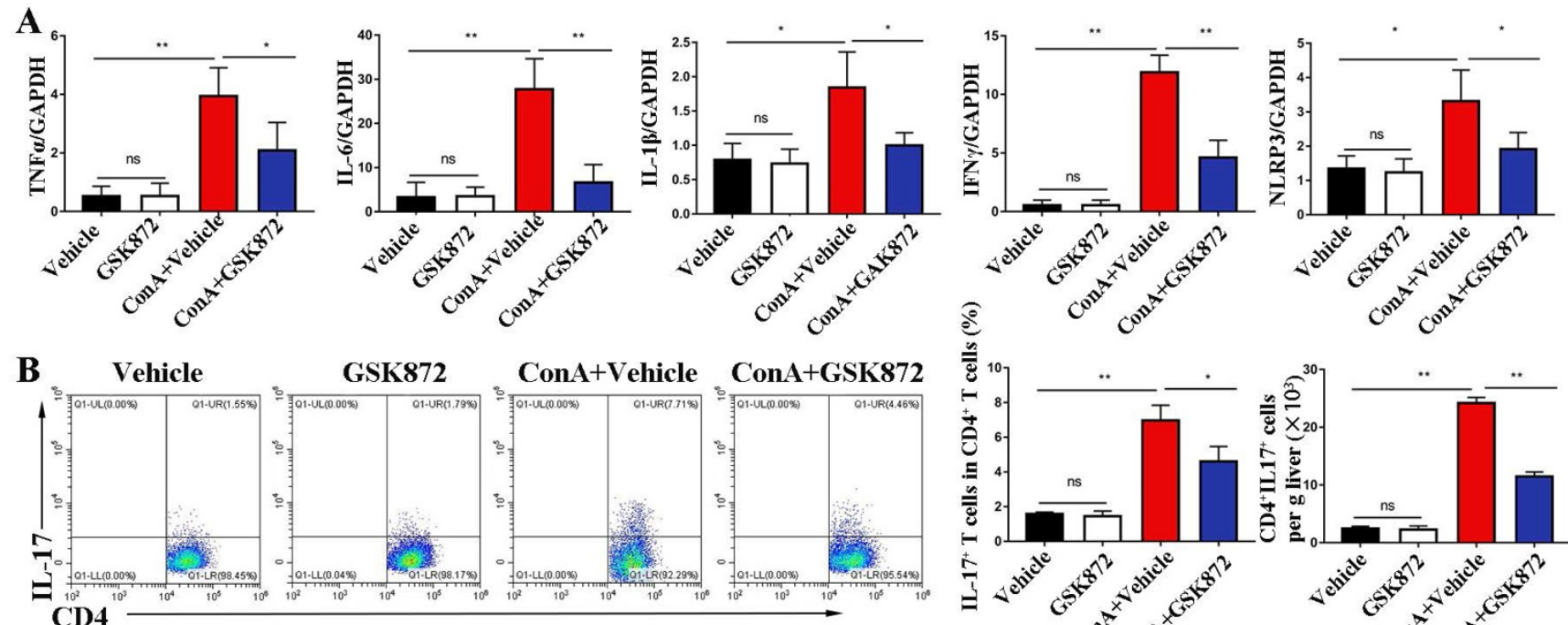

\section{GSK872}

ConA+Vehicle ConA+GSK872
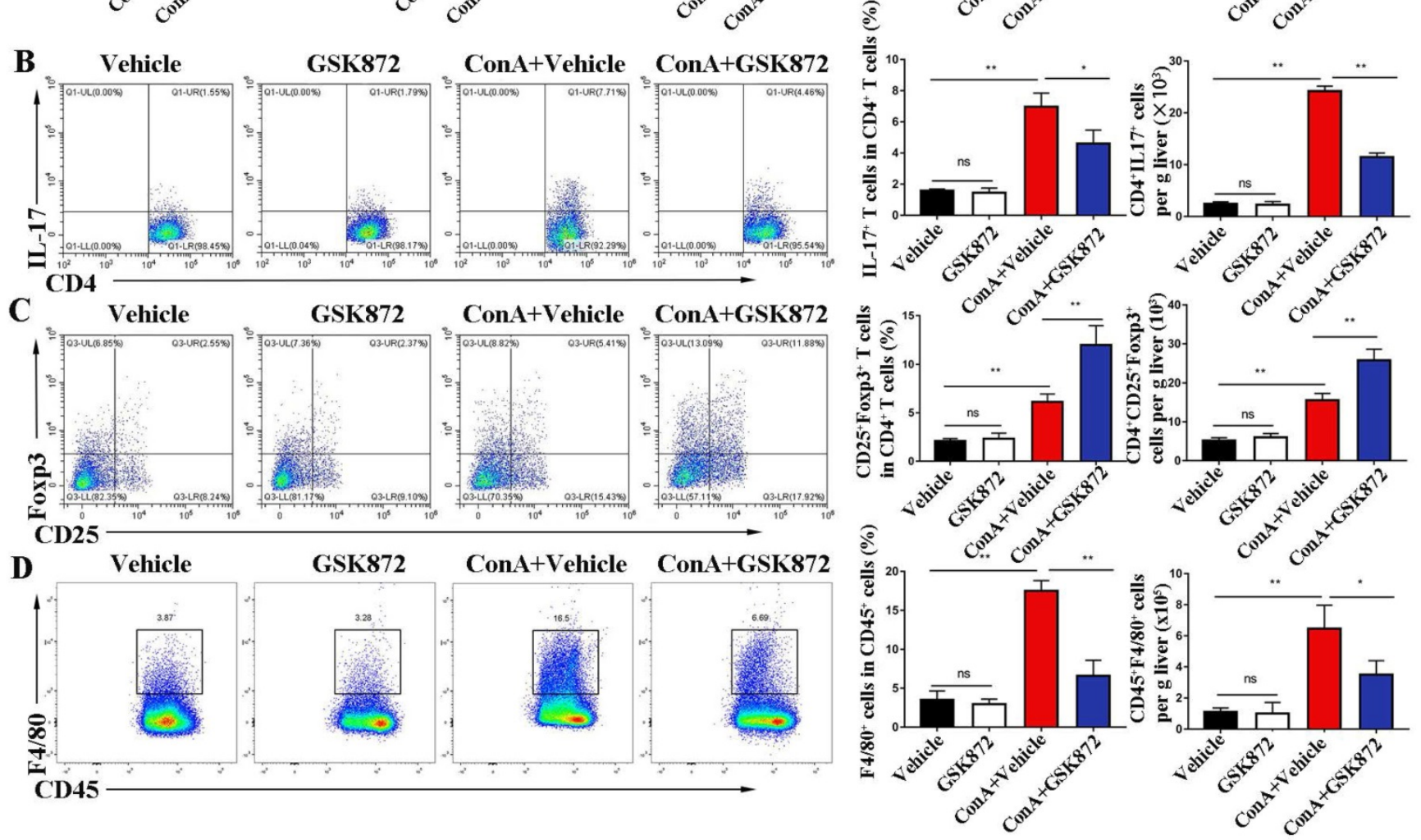

Figure 3. RIP3 blockade inhibits ConA-induced hepatic immune activation. (A) Expression levels of TNF- $\alpha$, IL-6, IL-1 $\beta$, IFNy, and NLRP3 in livers were analyzed by RT-qPCR. Gene expression was normalized to GAPDH levels. Fractions of CD4+IL-17+ cells (B) and CD4+CD25+ Foxp $3^{+}$cells (C) in liver CD4+ T cells were assessed by flow cytometry. Representative scatter plots (left) are presented, and the histogram (right) represents the statistical analysis of the percentages of gated in liver CD4+ $T$ cells. (D) Flow cytometry analysis of $\mathrm{CD}^{+} 5^{+} \mathrm{F} 4 / 80^{+}$macrophages in $\mathrm{CD} 45^{+} \mathrm{HMNCs}$ (left). The histogram (right) represents the statistical analysis of the percentages of $\mathrm{CD} 45^{+} \mathrm{F} 4 / 80^{+}$ macrophages in CD45+ HMNCs. All the values are shown as mean \pm SD. ns, not significant; $* P<0.05, * * p<0.01$. 

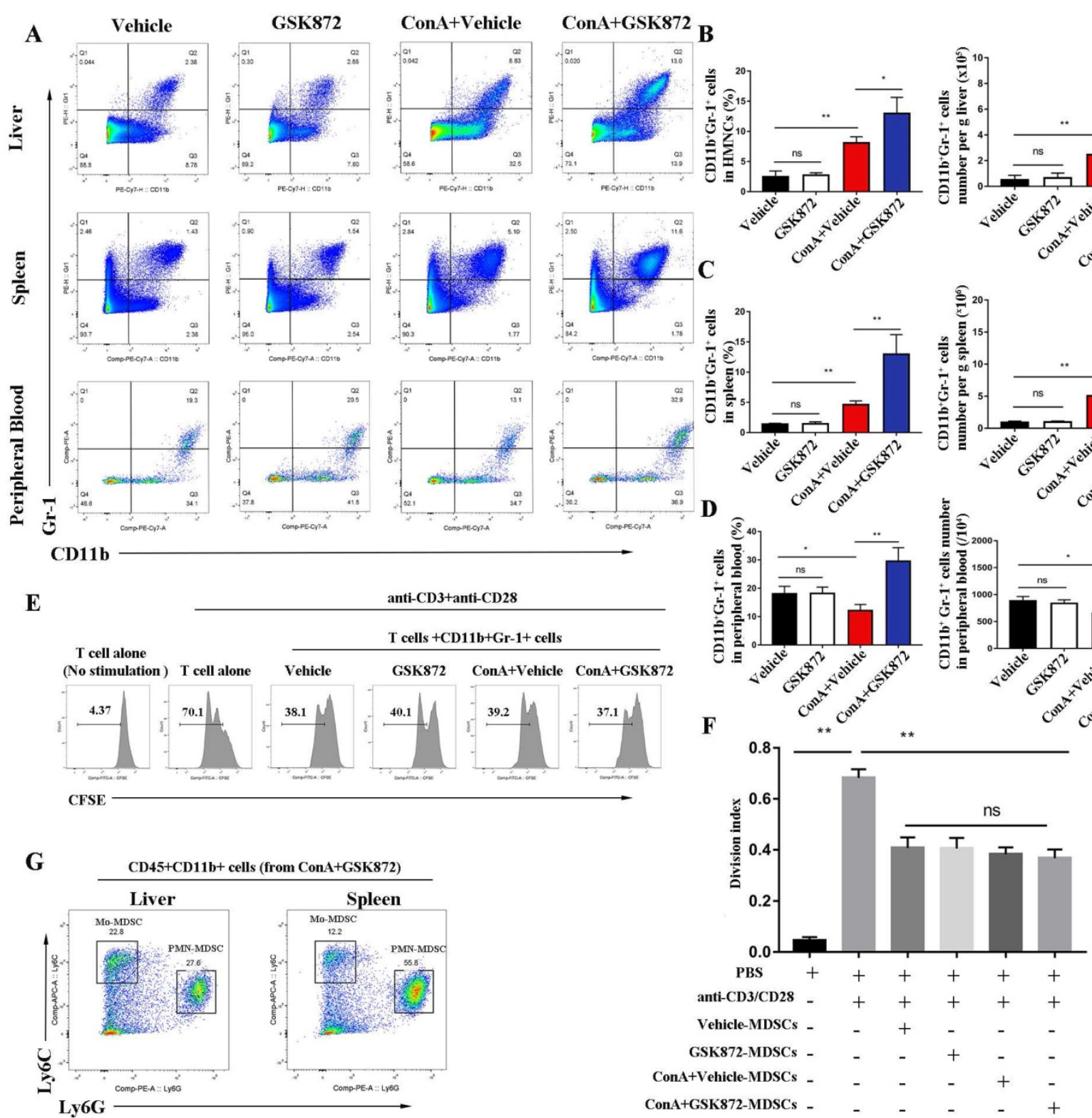

Figure 4. RIP3 blockade induces the accumulation of CDI Ib+Gr-1+ MDSCs in IMH mice. (A) Flow cytometry analysis of CDI Ib + Gr-1+ MDSCs in the liver, spleen, and peripheral blood. The statistical analysis of the percentages and absolute cell number of CD1 lb+Gr-1+MDSCs in the liver (B), spleen (C), and peripheral blood (D). (E) Flow cytometric analysis of proliferation of native splenic CD4 $4^{+} \mathrm{T}$ cell cocultured with diverse MDSCs in the presence of anti-CD3/CD28 for $72 \mathrm{~h}$. (F) The frequencies of the proliferating CD4+ T cells. (G) The percentage of Ly6GhighLy6Clo (PMN-MDSCs) and Ly6GloLy6Chi (Mo-MDSCs) in CD45+CD1 lb+ HMNCs of ConA+GSK872 treated-mice. All the values are shown as mean \pm SD. ns, not significant; $* P<0.05$, $* * P<0.01$.

Two major subsets of mice MDSCs have been identified based on the expression of CD11b, Ly6G and Ly6C antigens [18]. Polymorphonuclear MDSCs (PMN-MDSCs) expressing CD11b+Ly6G ${ }^{\text {hi }}$ Ly6Clo and monocytic MDSCs (Mo-MDSCs) expressing CD11b+Ly6GloLy6Chi. As shown in Figure 4G, both PMN-MDSCs (CD11b+Ly6G Gi $^{\text {Ly } 6 C^{\text {lo }}}$ ) and Mo-MDSCs $\left(\mathrm{CD} 11 b^{+}{ }^{2} y 6 \mathrm{G}^{\mathrm{l}} \mathrm{Ly} 6 \mathrm{C}^{\mathrm{hi}}\right)$ were present in the liver and spleen of mice treated with ConA+GSK872, and the majority of MDSCs were PMN-MDSCs.

\section{MDSCs mediate the hepatoprotective effect of GSK872}

Mice were given a depleting anti-Gr-1 antibody before GSK872 treatment to determine whether GSK872-mediated protection is mediated by MDSCs. The percentage of $\mathrm{CD} 11 \mathrm{~b}^{+} \mathrm{Gr}-1^{+}$MDSCs increased after treatment with GSK872 and ConA, but the cells were virtually absent in hepatic mononuclear cells (HMNCs) and spleen cells isolated from mice pretreated with anti-Gr-1 and GSK872 and then injected with ConA (Figure 5A). Furthermore, the percentage of both PMN-MDSCs and Mo-MDSCs (especially PMN-MDSCs) gated on $\mathrm{CD} 45^{+} \mathrm{CD} 11 \mathrm{~b}^{+}$ MDSCs were significantly reduced in livers and spleens after anti-Gr-1 treatment (Figure S2), and the secondary antibody staining (antibody-bound Gr-1 ${ }^{+}$ MDSCs) was almost undetectable in livers and 
spleens (not shown). These results demonstrated the ability of anti-Gr-1 treatment to effectively deplete MDSCs in livers and spleens. Analysis of serum transaminases (Figure 5B), spleen index (Figure 5C), H\&E staining of liver sections, and perusal images of livers and spleens (Figure 5D) showed that depletion of $\mathrm{Gr}-1^{+}$MDSCs was accompanied by the lack of GSK872-mediated protective effect against ConA-induced immune hepatitis.

In order to confirm the hepatoprotective effect of MDSCs, we performed MDSCs-depletion experiments in ConA treated-mice (Figure S3). The results showed that both $C D 11 b^{+} G r-1^{+}$MDSCs and its subtypes (PMN-MDSCs and Mo-MDSCs) were markedly reduced in livers of aGr1+ConA group compared $\mathrm{IgG}+$ ConA group. In addition, analysis of serum ALT/AST and H\&E staining of liver sections showed that depletion of $\mathrm{Gr}-1^{+}$MDSCs was accompanied by more severe liver damage, confirming the effect of MDSCs on protection of ConA-induced injury.
A Control
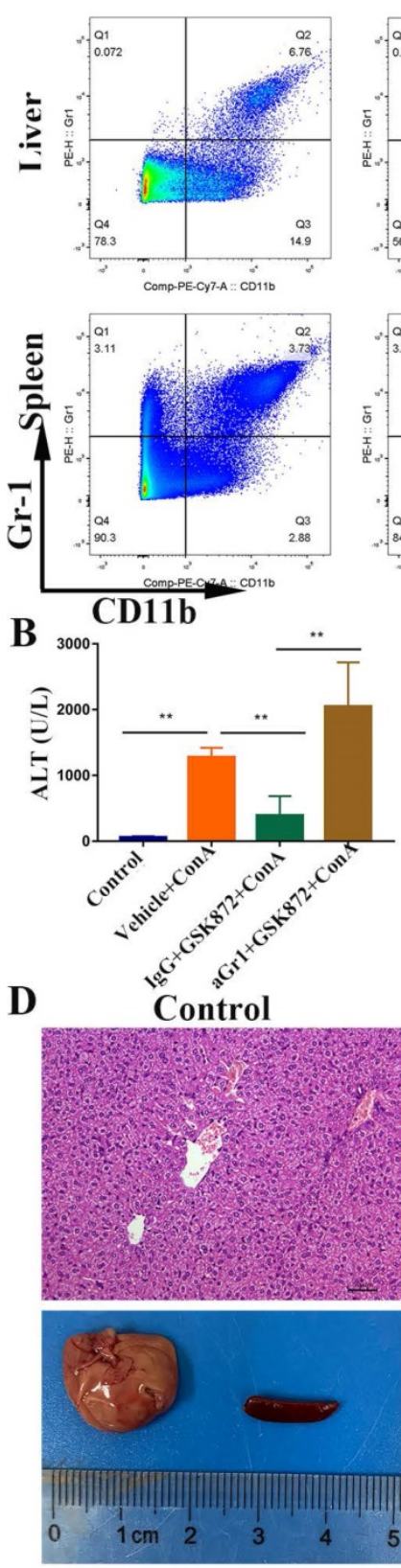

Vehicle+ConA
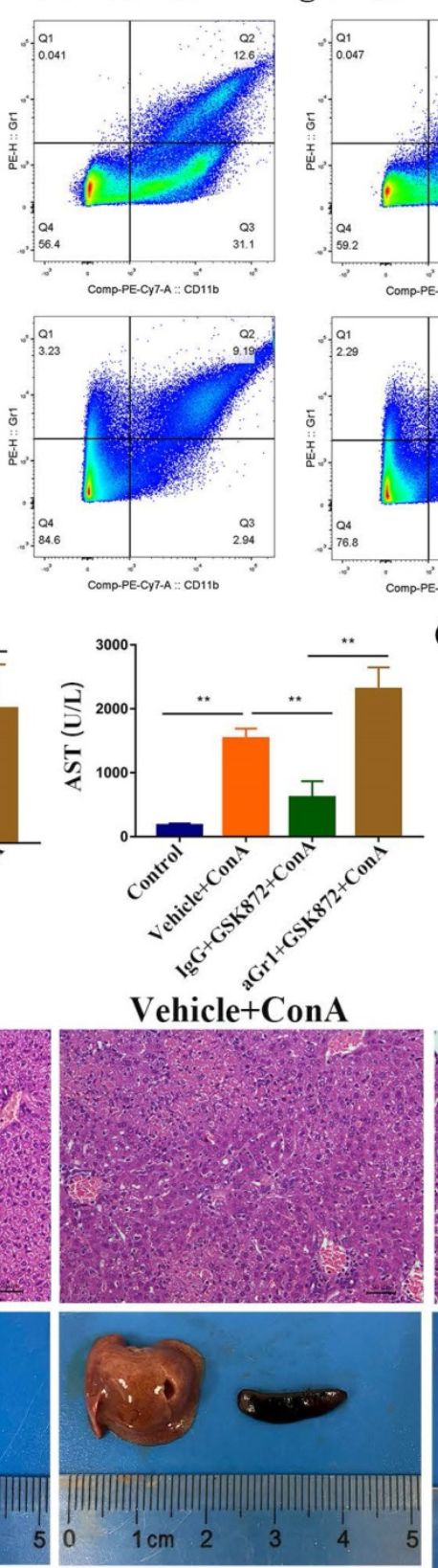
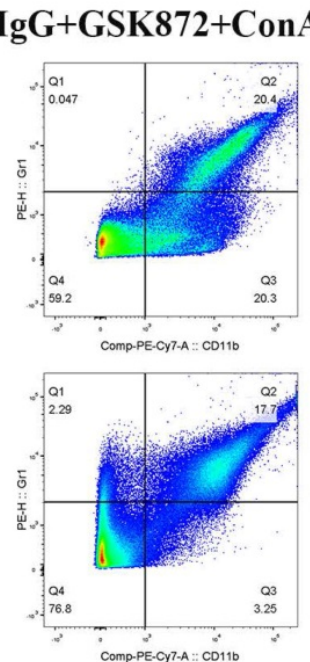

C

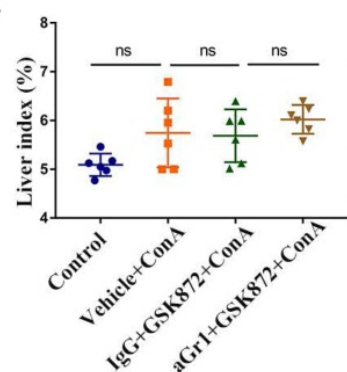

IgG+GSK872+ConA
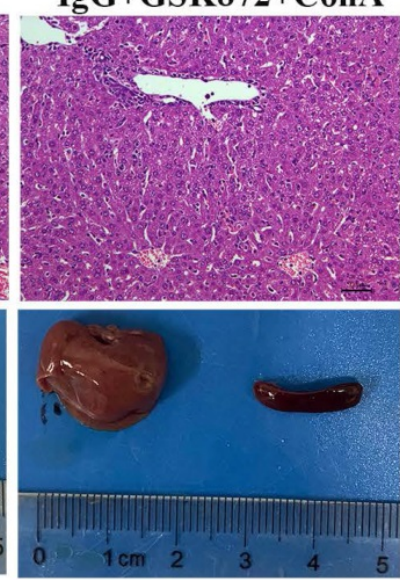

aGr1+GSK872+ConA
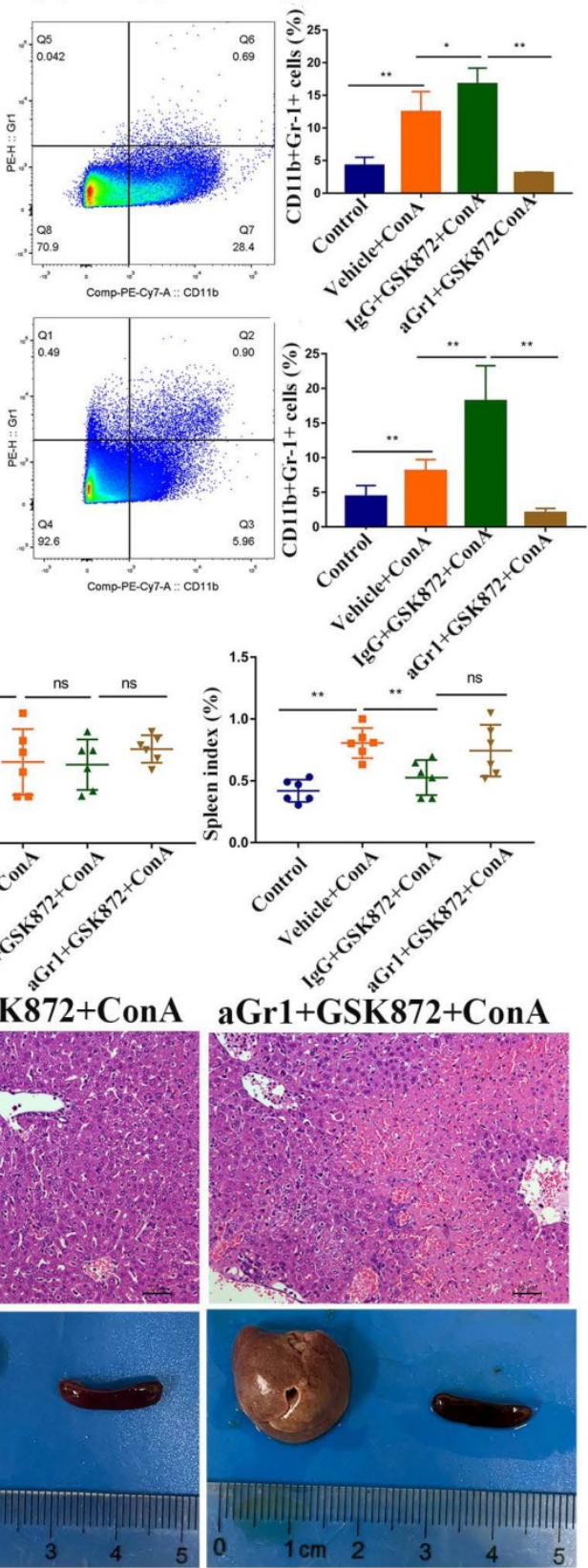

Figure 5. In vivo depletion of $\mathbf{G r}-1$ positive MDSCs abrogates $\mathbf{G S K 8 7 2 - m e d i a t e d ~ h e p a t o p r o t e c t i o n . ~ M i c e ~ w e r e ~ g i v e n ~ a n t i - G r - 1 ~ d e p l e t i n g ~ a n t i b o d y ~ ( ~} 250 \mu \mathrm{g})$ or control lgG $(250 \mu \mathrm{g})$, treated with GSK872 or vehicle $36 \mathrm{~h}$ later, and followed by administration of ConA or PBS ( $\mathrm{n}=6$ per group). (A) Flow cytometry analysis of CD $11 \mathrm{~b}+\mathrm{Gr}-1^{+}$ MDSCs in livers (up) and spleens (down). Representative scatter plots (left) are presented, and the histogram (right) represents the percentages of CD1 lb+Gr-1+ MDSCs. (B) Serum levels of ALT and AST were measured $12 \mathrm{~h}$ after ConA treatment. (C) Liver index (left) and spleen index (right) were determined $12 \mathrm{~h}$ after ConA treatment. (D) Representative photomicrographs (H\&E staining; original magnification 200x; scale bars, $50 \mu \mathrm{m}$; up) and perusal images (down) of livers. All the values are shown as mean \pm SD. ns., not significant; $* P<0.05, * * P<0.01$. 

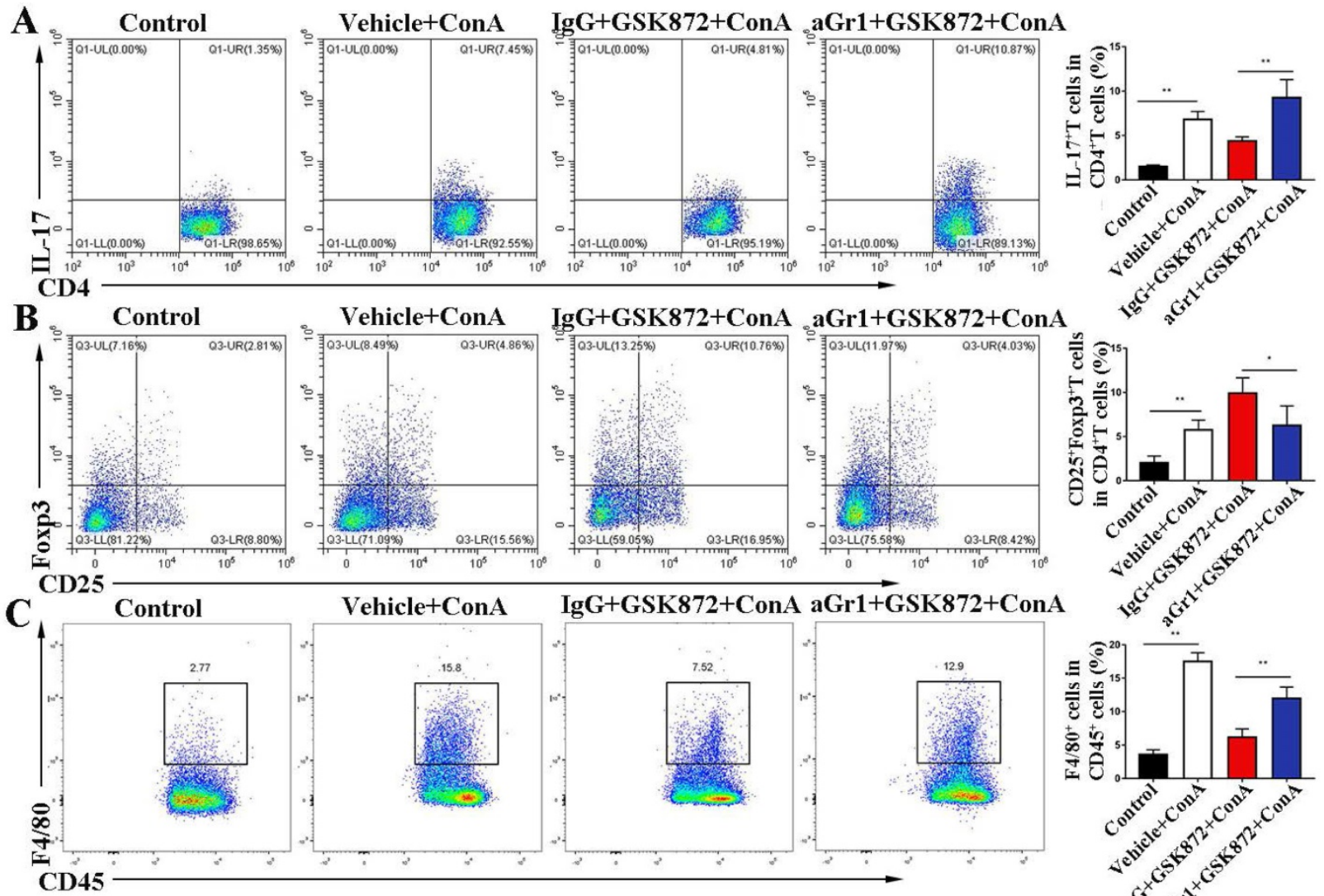

Vehicle+ConA
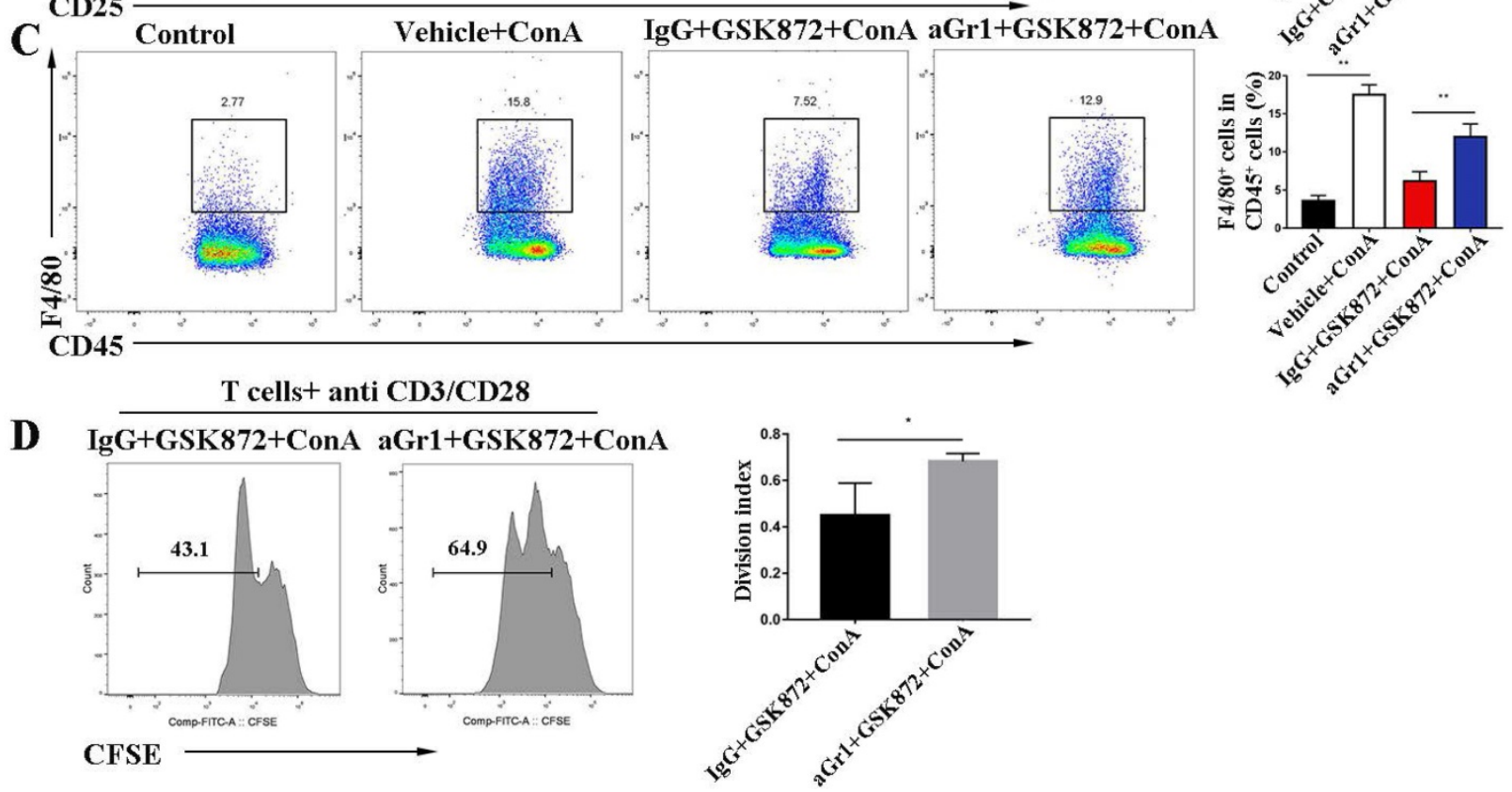

Figure 6. In vivo depletion of Gr-1 positive MDSCs attenuates GSK872-induced immunosuppression. Flow cytometry analysis of CD4+IL-17+ cells (A) and $\mathrm{CD}^{+} \mathrm{CD} 25^{+}$Foxp $3^{+}$cells (B) in livers. Representative scatter plots (left) are presented, and the histogram (right) represents the percentages of $\mathrm{CD}^{+} \mathrm{IL}-17^{+}$cells and $\mathrm{CD}^{+} \mathrm{CD} 25^{+}$Foxp $3^{+}$cells in liver CD4+ $\mathrm{T}$ cells. (C) Flow cytometry analysis of $\mathrm{CD} 45^{+} \mathrm{F} 4 / 80^{+}$macrophages in $\mathrm{CD}^{2} 5^{+} \mathrm{HMNCs}$ (left). The histogram (right) represents the statistical analysis of the percentages of CD45+F4/80+ macrophages in CD45+ HMNCs. (D) Splenic CD4+ $\mathrm{T}$ cells isolated from IgG+GSK872+ConA and aGrl+ GSK872+ConA treated-mice were cultured in the presence of anti-CD3/CD28 for $72 \mathrm{~h}$. Flow cytometric analysis (left) and the statistical analysis (right) of the proliferation of CD4+ T cells. All the values are shown as mean \pm SD. ns, not significant; $* P<0.05$, $* * P<0.01$.

\section{GSK872-induced anti-inflammatory effect requires the involvement of MDSCs}

To investigate the mechanism by which $\mathrm{Gr}-1^{+}$ MDSC deletion abrogates the hepatoprotective effect mediated by GSK872, we examined the proinflammatory cells involved in IMH. Flow cytometry analysis showed that ConA-induced liver accumulation of $\mathrm{CD} 4^{+} \mathrm{IL}-17^{+}$Th17 cells (Figure 6A) and $\mathrm{CD} 45^{+} \mathrm{F} 4 / 80^{+}$macrophages (Figure $6 \mathrm{C}$ ) was markedly reduced in mice pretreatment with GSK872. However, the depletion of Gr-1+ MDSCs dramatically reversed the GSK872-mediated low infiltration of CD4+IL-17+Th17 cells and CD45 ${ }^{+} \mathrm{F} 4 / 80^{+}$macrophages and decreased the percentage of $\mathrm{CD} 4^{+} \mathrm{CD} 25^{+} \mathrm{Foxp} 3^{+}$ Tregs (Figure 6B). Furthermore, we isolated $\mathrm{CD}^{+} \mathrm{T}$ cells from the spleen of IgG+GSK872+ConA treatedor aGr1+GSK872+ConA treated-mice and analyzed their proliferation ability after stimulation with anti-CD3/CD28 for 3 days (Figure 6D). The results showed that anti-Gr-1 increased the proliferation ability of splenic $\mathrm{CD}^{+}{ }^{+} \mathrm{T}$ cells compared to IgG control, indicating that MDSCs depletion promotes the activation of $\mathrm{T}$ cells. Thus, all these data indicate that MDSCs mediate GSK872-induced antiinflammatory effect and immunosuppressive environment in livers of IMH mice. 
A
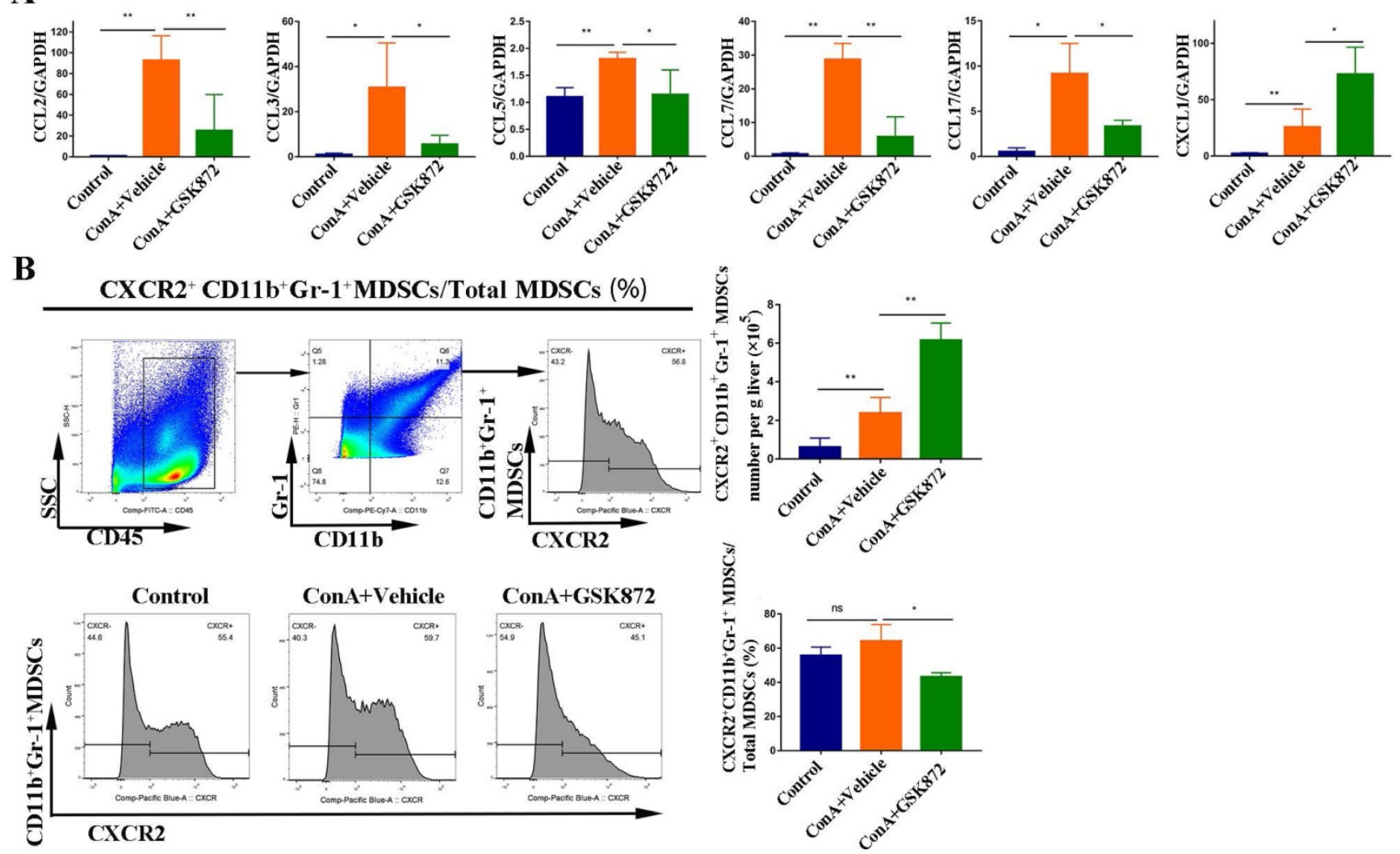

Figure 7. GSK872 induces CXCL1 in the livers of ConA-treated mice. Mice were given GSK872 or vehicle i.p. $1 \mathrm{~h}$ before treatment with ConA or PBS and sacrificed $12 \mathrm{~h}$ after ConA treatment. Livers were explanted and used for RNA and mononuclear cell extraction. (A) Gene expression levels of CCL2, CCL3, CCL5, CCL7, CCL17, and CXCL1 in liver tissues from mice treated with ConA and GSK872 were analyzed using RT-qPCR. Gene expression was normalized to GAPDH levels. (B) Flow cytometry analysis of the absolute cell number and percentage of CXCR2+CD11 b+Gr+ MDSCs in livers. Gating strategies for CXCR2+CD11 b+Gr-1+ MDSCs are shown (up). The histogram (right) represents the statistical analysis of the $d$ absolute cell number of CXCR2 $2^{+} C D 11 b^{+} G r^{+}$MDSCs in livers and the percentages of CXCR2 ${ }^{+} C D 11 b^{+} G r-1^{+} M D S C s$ in total liver CD 11 b+Gr-1+ MDSCs. Representative histograms in flow cytometry analysis (down) are presented. All the values are shown as mean \pm SD. ns., not significant; $* P<0.05, * * P<$ 0.01 .

\section{RIP3 blockade induces the production of chemokine (C-X-C motif) ligand 1 (CXCL1) in the liver}

Chemotaxis and their cognate receptors play a crucial role in the migration of MDSCs into tissues [40]; therefore, we identified the gene expression of several chemokines regulated by RIP3 inhibition (Figure 7A). The expressions of $\mathrm{C}-\mathrm{C}$ motif chemokine ligand (CCL)2, CCL3, CCL5, CCL7, and CCL17 all increased in the livers of mice treated with ConA but were markedly decreased by pretreatment with GSK872. By contrast, induction of IMH by ConA was accompanied by a significant upregulation of CXCL1 mRNA, and it was further increased by pretreatment with GSK872, consistent with the changes in the percentage of hepatic CD11 $b^{+} \mathrm{Gr}-1^{+}$MDSCs.

To further delineate the possible crosstalk between CXCL1 and MDSCs, we next used flow cytometry to investigate the percentages of MDSCs expressing CXCR2, the cognate receptor for CXCL1. ConA significantly increased the absolute cell number of hepatic CD11 b+Gr-1+ MDSCs expressing CXCR2 compared with the control mice. GSK872 further enhanced the absolute cell number of hepatic $\mathrm{CD} 11 \mathrm{~b}^{+} \mathrm{Gr}-1^{+}$MDSCs expressing CXCR2 induced by ConA (Figure 7B), consistent with the changes in the

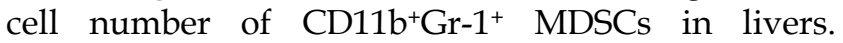
Moreover, $\mathrm{CD} 11 \mathrm{~b}^{+} \mathrm{Gr}-1^{+}$MDSCs isolated from HMNCs of the three groups all expressed a high level of CXCR2, indicating that CXCL1-CXCR2 axis contributes to MDSCs recruitment induced by RIP3 inhibition. Interestingly, although GSK872 further enhanced the cell number of hepatic CXCR2 ${ }^{+} \mathrm{CD} 11 \mathrm{~b}^{+}$ Gr- $1^{+}$MDSCs induced by ConA, the ratio of CXCR2 ${ }^{+}$ $\mathrm{CD} 11 \mathrm{~b}+\mathrm{Gr}-1^{+}$MDSCs to total CD11b+Gr-1+ ${ }^{+}$MDSCs slightly reduced, suggesting that the CXCL1-CXCR2 axis is an important but not the only cause of the accumulation of liver MDSCs mediated by RIP3 inhibition.

\section{Discussion}

The present study indicates that RIP3 blockade and subsequent MDSCs accumulation protected mice from ConA-induced immune hepatitis. RIP3 blockade reduced the production of proinflammatory cytokines 
and the infiltration of $\mathrm{CD} 4^{+} \mathrm{IL}-17^{+}$Th17 cells and $\mathrm{CD} 45^{+} \mathrm{F} 4 / 80^{+}$macrophages through a MDSCdependent mechanism, thus attenuating liver immune activity. Moreover, we showed that RIP3 inhibition may be a novel mechanism underlying the immunosuppressive effect of glucocorticoids on AIH. Therefore, RIP3 signaling can be a valuable therapeutic target in IMH.

RIP3, a member of the RIP kinase family, is a key inflammatory adapter and has critical roles in innate inflammation and immunoreaction through necroptosis and non-necroptosis function [41-44]. The upregulation of RIP3 expression has been described in $\mathrm{AIH}$ and other immune disorders and supposed to contribute to the progression of the pathology $[45,46]$. Zhang et al. [31] showed that the accumulated macrophages express RIP3 in AIH liver tissues. And RIP3 deletion has been reported to protect against ConA-induced immune hepatitis [47]. However, no investigation has been reported on the activation of RIP3 signaling and its role in liver inflammation during AIH development. In this study, we indicated that RIP3 and its effector molecule MLKL were activated in livers of $\mathrm{AIH}$ patients and ConA-induced immune hepatitis mice, and these expressions were localized in the majority of the macrophages (Kupffer cells). Blockading RIP3 signaling with GSK872, a specific RIP3 kinase inhibitor, prevented ConAinduced immune hepatitis and reduced the levels of transaminases and liver inflammation. Moreover, RIP3 signaling was demonstrated as a novel molecular mechanism of DEX treatment. Overall, these data indicate that targeting RIP3 signaling may be a valuable therapeutic strategy for the prevention of AIH.

Given that numerous immune cells (e.g., T cells, $B$ cells, and macrophages) and cytokines are involved in the pathogenesis of $\mathrm{AIH}$, we next explored the effect of RIP3 blockade on the production of proinflammatory cytokines and infiltration of immune cells in the livers of ConA-treated mice. Induction of immune hepatitis induced by ConA was followed by a significant upregulation of TNFa, IL-6, IL-1 $\beta$, NLRP3, and IFN $\gamma$ in liver tissues, whereas pretreatment of animals with GSK872 before administration of ConA caused a significant decrease

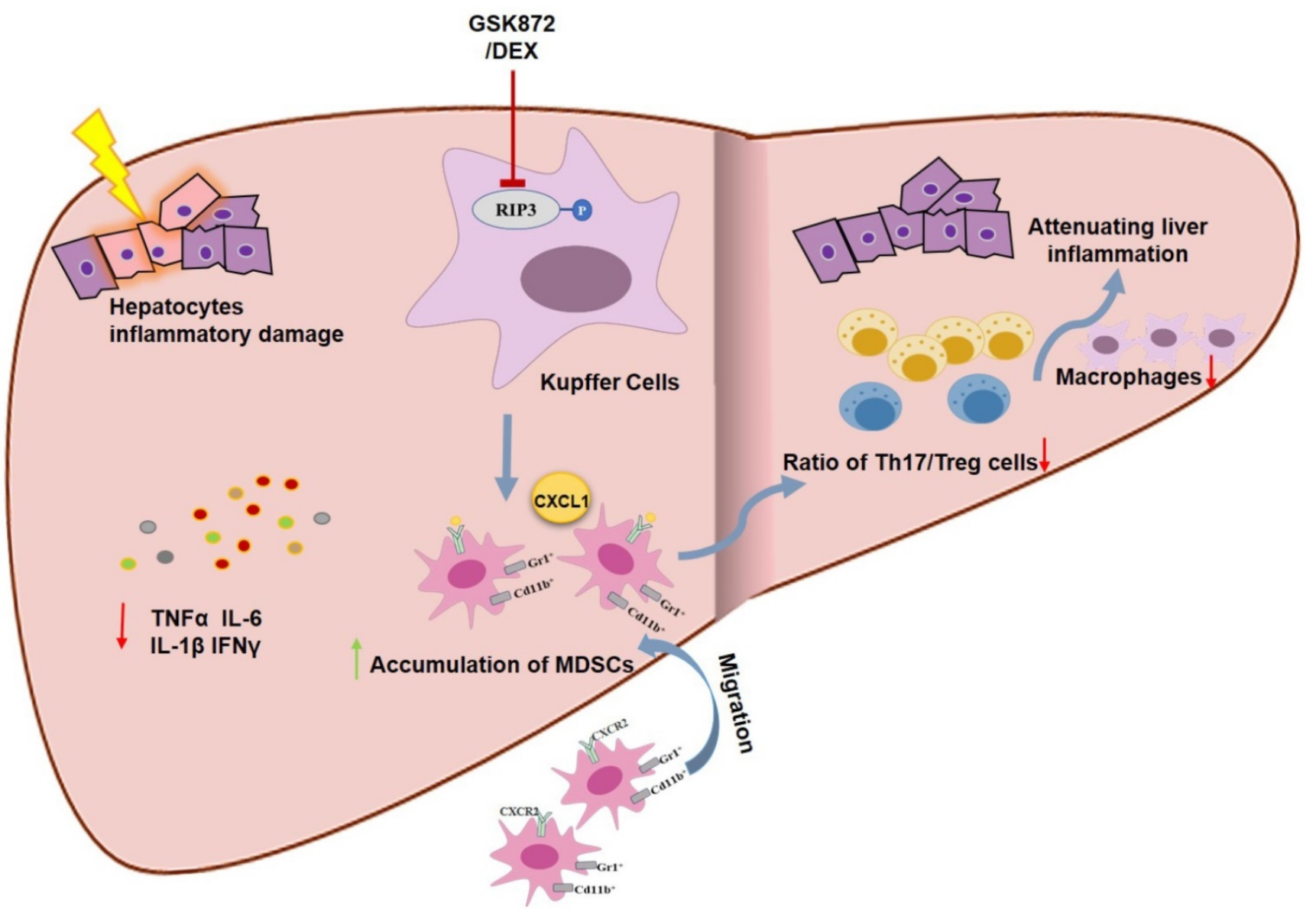

Figure 8. Schematic representation of RIP3 blockade in preventing immune-mediated hepatitis. RIP3 kinase inhibition promotes the hepatic accumulation of MDSCs by CXCL1-CXCR2 axis and other unknown factors. Accumulated MDSCs mediate the hepatoprotective effect of GSK872 by reducing the frequencies of Th17 cells and macrophages but increasing those of Treg cells. 
in the expression of these proinflammatory cytokines. These cytokines are induced in $\mathrm{AIH}$ and contribute to the related detrimental immune reactions [27, 48]. Moreover, we observed that RIP3 blockade modified the type of immune cells infiltrating the liver of IMH mice, decreasing the frequencies of $\mathrm{CD}^{+} \mathrm{IL}-17^{+} \mathrm{Th} 17$ cells and $\mathrm{CD} 45^{+} \mathrm{F} 4 / 80^{+}$macrophages but increasing those of $\mathrm{CD}_{4}{ }^{+} \mathrm{CD} 25^{+} \mathrm{Foxp}^{+}$Tregs. The imbalance between Th17 and Tregs contributes to the induction and perpetuation of liver injury in $\mathrm{AIH}[12,49]$. In addition, macrophages are present in dense portal cell infiltrates of AIH [50], whereas macrophage activation contributes to the inflammation and fibrosis in liver diseases [51, 52]. These results show that RIP3 blockade attenuates liver immune activity response to ConA.

To dissect the mechanism of RIP3 blockade in regulating hepatic inflammatory reactions, we investigated the infiltration of MDSCs with significant immune inhibitory function in mice with IMH with or without GSK872 treatment. MDSCs have been implicated in the downregulation of immune responses through inhibiting the activation and proliferation of T cells, B cells, macrophages, and NK cells and reducing the production of proinflammatory factors [23, 53, 54]. Diao et al. [27] and Liu et al. [54] demonstrated that the frequencies of MDSCs in mouse spleen and liver were increased at early stage of ConA treatment, MDSCs adoptive transfer protected mice from ConA-induced hepatitis, suggesting that the MDSCs might be involved in inhibiting early acute inflammation caused by ConA, increasing the frequency of MDSCs may be a novel strategy in suppressing liver inflammation. Consistent with these findings, we observed that MDSCs were expanded in the liver and spleen of ConA-induced mice, but reduced in the peripheral blood, implicating that the migration of periphery MDSCs to the liver and spleen may be involved in the initial resistance of mice against IMH. More importantly, we showed that RIP3 blockade can further promote the accumulation of MDSCs in liver, spleen, and periphery of mice with IMH. However, GSK872 by itself was not able to modify the percentage of MDSCs in liver and spleen of mice without hepatitis, it is not surprising. Because, it has been reported that inflammation is required for induction of MDSCs $[55,56]$. Although CD11b and Gr-1 are surface markers common to neutrophils and MDSCs, the immunosuppressive properties of MDSCs allow them to be distinguished from former [57]. Hepatic MDSCs isolated from ConA+GSK872 and other group inhibited the proliferation of activated $\mathrm{T}$ cells in vitro, thus confirming their suppressive nature. Overall, these findings suggest that the immunosuppressive effect of RIP3 blockade may be associated with MDSCs expansion. To test the hypothesis, we evaluated the effect of MDSCs depletion on the effect of RIP3 blockade on the ConA-induced IMH. The depletion of MDSCs from mice abolished the GSK872-mediated protection against immune mediated liver damage and abrogated the GSK872-induced hepatic immune tolerance in IMH mice. Thus, RIP3 blockade protected mice from ConA-induced immune hepatitis through MDSC-mediated immune tolerance.

The underling mechanism by which GSK872 promotes accumulation of MDSCs in mice with IMH remains to be ascertained. Chemokines, including CCL2, CCL17, and CXCL1, play a crucial role in the recruitment of MDSCs into the tissues through their cognate receptors $[37,40]$. In this study, we showed that of all the chemokines evaluated, CXCL1 was one of the most abundantly expressed in ConA-treated mice and was further enhanced by RIP3 blockade, suggested that the expression of CXCL1 may be regulated by RIP3 kinase. The work of $\mathrm{Li}$ et al[58] supports our assumption, they reported that RIP3 deficiency induced nuclear translocation of p-P65Ser536 and the binding of p-P65Ser536 to the CXCL1 promoter increased the transcription of CXCL1. By contrast, the expressions of CCL2, CCL3, CCL5, CCL7, and CCL17 increased in the livers of mice treated with ConA but were downregulated by GSK872 treatment. Consistently, $\mathrm{CD} 11 \mathrm{~b}^{+} \mathrm{Gr} 1^{+}$MDSCs isolated from livers of mice with or without ConA treatment expressed high levels of CXCR2, the CXCL1 cognate receptor. Although GSK872 further enhanced the cell number of hepatic $\mathrm{CXCR} 2{ }^{+} \mathrm{CD} 11 \mathrm{~b}+\mathrm{Gr}-1^{+}$MDSCs induced by ConA, the ratio of $\mathrm{CXCR} 2{ }^{+} \mathrm{CD} 11 \mathrm{~b}^{+} \mathrm{Gr}-1^{+}$MDSCs to total $\mathrm{CD} 11 \mathrm{~b}^{+} \mathrm{Gr}-1^{+}$MDSCs slightly reduced, suggesting that the CXCL1-CXCR2 axis is an important but not the only cause of the accumulation of liver MDSCs mediated by RIP3 inhibition. Further studies are required to determine whether RIP3 inhibition converts tissue resident cells into MDSCs.

In conclusion, our study demonstrated for the first time that MDSCs accumulation derived by RIP3 blockade can effectively protect mice from ConA-induced IMH through downregulating hepatic inflammatory responses, raising the possibility that RIP3 blockade can be a promising new therapeutic approach for treating AIH.

\section{Abbreviations}

AIH: autoimmune hepatitis; RIP3: receptor interacting protein kinase 3; ConA: concanavalin A; MDSCs: myeloid-derived suppressor cells; Tregs: $\mathrm{T}$ regulatory cells; NK: natural killer; IMH: immune-mediated hepatitis; TNF: tumor necrosis 
factor; MLKL: mixed lineage kinase domain like protein; IL: interleukin; NLRP3: NLR family pyrin domain containing 3; IFN: interferon; iNOS: inducible nitric oxide synthase; CCL: C-C motif chemokine ligand; i.v.: intravenously; i.p.: intraperitoneally; DEX: dexamethasone; PBS: phosphate-buffered saline; ALT: alanine aminotransferase; AST: aspartate aminotransferase; HMNCs: hepatic mononuclear cells; CXCL1: chemokine (C-X-C motif) ligand 1; CXCR2: chemokine (C-X-C motif) receptor 2; FITC: fluorescein isothiocyanate; SD: standard deviation.

\section{Supplementary Material}

Supplementary figures.

https://www.ijbs.com/v18p0199s1.pdf

\section{Acknowledgements}

This work was supported by grants from the National Natural Science Foundation of China (Grant No. 81860109). The funders had no role in study design, data collection, data analysis, interpretation, writing and revision of the manuscript.

\section{Author Contributions}

L.Zhou., JW.Z. and BM.W. designed the study. M.L., HX.Z. and L.Zhang. performed the experiments. M.L, HX.Z., L.Zhang., X.L., SM.Z., XX.W. and WL.Z. analyzed the results. M.L. and L.Zhou. wrote the paper. L.Zhou., JW.Z., BM.W. and J.Z. directed the research and made the critical revision. M.L., HX.Z. and L.Zhang. contributed equally to this work. All authors contributed to the article, approved the submitted version.

\section{Data Availability}

The datasets generated during and/or analyzed during the current study are available from the corresponding author on reasonable request.

\section{Competing Interests}

The authors have declared that no competing interest exists.

\section{References}

1. Mieli-Vergani G, Heller S, Jara P, et al. Autoimmune hepatitis. J Pediatr Gastroenterol Nutr. 2009; 49: 158-64.

2. Manns MP, Czaja AJ, Gorham JD, et al. Diagnosis and management of autoimmune hepatitis. Hepatology. 2010; 51: 2193-213.

3. Lohse AW, Mieli-Vergani G. Autoimmune hepatitis. J Hepatol. 2011; 55: 171-82.

4. Webb GJ, Hirschfield GM, Krawitt EL, et al. Cellular and Molecular Mechanisms of Autoimmune Hepatitis. Annu Rev Pathol. 2018; 13: 247-92.

5. Baven-Pronk M, Biewenga M, van Silfhout JJ, et al. Role of age in presentation, response to therapy and outcome of autoimmune hepatitis. Clin Transl Gastroenterol. 2018; 9: 165.

6. Summerskill $\mathrm{WH}$, Korman MG, Ammon HV et al. Prednisone for chronic active liver disease: dose titration, standard dose, and combination with azathioprine compared. Gut. 1975; 16: 876-83.

7. Schäcke H, Döcke WD, Asadullah K. Mechanisms involved in the side effects of glucocorticoids. Pharmacol Ther. 2002; 96: 23-43.
8. Manns MP, Woynarowski M, Kreisel W, et al. Budesonide induces remission more effectively than prednisone in a controlled trial of patients with autoimmune hepatitis. Gastroenterology. 2010; 139: 1198-206.

9. Friedman SL. Mechanisms of hepatic fibrogenesis. Gastroenterology. 2008; 134: 1655-69.

10. Longhi MS, Mitry RR, Samyn M, et al. Vigorous activation of monocytes in juvenile autoimmune liver disease escapes the control of regulatory T-cells. Hepatology. 2009; 50: 130-42.

11. Sunami Y, Leithäuser F, Gul S, et al. Hepatic activation of IKK/NFKB signaling induces liver fibrosis via macrophage-mediated chronic inflammation. Hepatology. 2012; 56: 1117-28.

12. Liberal R, Grant CR, Yuksel M, et al. Regulatory T-cell conditioning endows activated effector $\mathrm{T}$ cells with suppressor function in autoimmune hepatitis/ autoimmune sclerosing cholangitis. Hepatology. 2017; 66: 1570-84.

13. Tiegs G, Hentschel J, Wendel A. A T cell-dependent experimental liver injury in mice inducible by concanavalin A. J Clin Invest. 1992; 90: 196-203.

14. Gantner F, Leist M, Lohse AW, et al. Concanavalin A-induced T-cell-mediated hepatic injury in mice: the role of tumor necrosis factor. Hepatology. 1995; 21: $190-8$

15. He GW, Günther C, Kremer AE, et al. PGAM5-mediated programmed necrosis of hepatocytes drives acute liver injury. Gut. 2017; 66: 716-23.

16. Knolle PA, Gerken G. Local control of the immune response in the liver. Immunol Rev. 2000; 174: 21-34.

17. Seki E, Brenner DA. Toll-like receptors and adaptor molecules in liver disease: update. Hepatology. 2008; 48: 322-35.

18. Bronte V, Brandau S, Chen SH, et al. Recommendations for myeloid-derived suppressor cell nomenclature and characterization standards. Nat Commun. 2016; 7: 12150

19. Gabrilovich DI. Myeloid-Derived Suppressor Cells. Cancer Immunol Res. 2017; 5: 3-8.

20. Neamah WH, Singh NP, Alghetaa $H$, et al. AhR Activation Leads to Massive Mobilization of Myeloid-Derived Suppressor Cells with Immunosuppressive Activity through Regulation of CXCR2 and MicroRNA miR-150-5p and miR-543-3p That Target Anti-Inflammatory Genes. J Immunol. 2019; 203: $1830-44$

21. Turley SJ, Cremasco V, Astarita JL. Immunological hallmarks of stromal cells in the tumour microenvironment. Nat Rev Immunol. 2015; 15: 669-82.

22. Li J, Lee Y, Li Y, et al. Co-inhibitory Molecule B7 Superfamily Member 1 Expressed by Tumor-Infiltrating Myeloid Cells Induces Dysfunction of Anti-tumor CD8+ T Cells. Immunity. 2018; 48: 773-86.e5.

23. Veglia F, Perego M, Gabrilovich D. Myeloid-derived suppressor cells coming of age. Nat Immunol. 2018; 19: 108-19.

24. Kodach LL, Peppelenbosch MP. Targeting the Myeloid-Derived Suppressor Cell Compartment for Inducing Responsiveness to Immune Checkpoint Blockade Is Best Limited to Specific Subtypes of Gastric Cancers. Gastroenterology. 2021; 161: 727.

25. Oh MH, Sun $\mathrm{IH}$, Zhao L, et al. Targeting glutamine metabolism enhances tumor-specific immunity by modulating suppressive myeloid cells. J Clin Invest. 2020; 130: 3865-84

26. Buxbaum NP. We didn't start the fire, MDSC inflammasome signaling in GVHD. Blood. 2019; 134: 1570-2.

27. Diao $\mathrm{W}$, Jin $\mathrm{F}$, Wang $\mathrm{B}$, et al. The protective role of myeloid-derived suppressor cells in concanavalin A-induced hepatic injury. Protein Cell. 2014; 5: 714-24.

28. Zheng W, Wang $\mathrm{Q}, \mathrm{Lu} X$, et al. Protective Effects of Dracocephalum heterophyllum in ConA-Induced Acute Hepatitis. Mediators Inflamm. 2016; 2016: 2684321

29. Zheng W, Du S, Tian M, et al. Lepidium meyenii Walp Exhibits Anti-Inflammatory Activity against ConA-Induced Acute Hepatitis. Mediators Inflamm. 2018; 2018: 8982756.

30. Zhang DW, Shao J, Lin J, et al. RIP3, an energy metabolism regulator that switches TNF-induced cell death from apoptosis to necrosis. Science. 2009; 325: 332-6.

31. Zhang J, Guo L, Liu M, et al. Receptor-interacting protein kinase 3 mediates macrophage/monocyte activation in autoimmune hepatitis and regulates interleukin-6 production. United European Gastroenterol J. 2018; 6: 719-28.

32. Seifert L, Werba G, Tiwari S, et al. The necrosome promotes pancreatic oncogenesis via CXCL1 and Mincle-induced immune suppression. Nature. 2016; 532: 245-9.

33. Wang W, Marinis JM, Beal AM, et al. RIP1 Kinase Drives MacrophageMediated Adaptive Immune Tolerance in Pancreatic Cancer. Cancer Cell. 2018; 34: 757-74.e7.

34. Alvarez F, Berg PA, Bianchi FB, et al. International Autoimmune Hepatitis Group Report: review of criteria for diagnosis of autoimmune hepatitis. J Hepatol. 1999; 31: 929-38.

35. Shan $Z$, Liu $X$, Chen $Y$, et al. Chitinase 3-like-1 promotes intrahepatic activation of coagulation through induction of tissue factor in mice. Hepatology. 2018; 67: 2384-96.

36. Ye T, Wang T, Yang $X$, et al. Comparison of Concanavalin a-Induced Murine Autoimmune Hepatitis Models. Cell Physiol Biochem. 2018; 46: 1241-51.

37. Sarra M, Cupi ML, Bernardini R, et al. IL-25 prevents and cures fulminant hepatitis in mice through a myeloid-derived suppressor cell-dependent mechanism. Hepatology. 2013; 58: 1436-50. 
38. Condamine $\mathrm{T}$, Kumar V, Ramachandran IR, et al. ER stress regulates myeloid-derived suppressor cell fate through TRAIL-R-mediated apoptosis. J Clin Invest. 2014; 124: 2626-39.

39. Condamine T, Gabrilovich DI. Molecular mechanisms regulating myeloid-derived suppressor cell differentiation and function. Trends Immunol. 2011; 32: 19-25.

40. Chiu DK, Xu IM, Lai RK, et al. Hypoxia induces myeloid-derived suppressor cell recruitment to hepatocellular carcinoma through chemokine (C-C motif) ligand 26. Hepatology. 2016; 64: 797-813.

41. Kaczmarek A, Vandenabeele P, Krysko DV. Necroptosis: the release of damage-associated molecular patterns and its physiological relevance. Immunity. 2013; 38: 209-23.

42. Moriwaki K, Chan FK. Necrosis-dependent and independent signaling of the RIP kinases in inflammation. Cytokine Growth Factor Rev. 2014; 25: 167-74.

43. Moriwaki K, Chan FK. Necroptosis-independent signaling by the RIP kinases in inflammation. Cell Mol Life Sci. 2016; 73: 2325-34.

44. He S, Wang L, Miao L, et al. Receptor interacting protein kinase-3 determines cellular necrotic response to TNF-alpha. Cell. 2009; 137: 1100-11.

45. Lin J, Li H, Yang M, et al. A role of RIP3-mediated macrophage necrosis in atherosclerosis development. Cell Rep. 2013; 3: 200-10.

46. Chaohuan G, Rong F, Mianjing Z, et al. Pathogenesis of lupus nephritis: RIP3 dependent necroptosis and NLRP3 inflammasome activation. J Autoimmun. 2019; 103 .

47. Deutsch M, Graffeo CS, Rokosh R, et al. Divergent effects of RIP1 or RIP3 blockade in murine models of acute liver injury. Cell Death Dis. 2015; 6: e1759.

48. Luan J, Zhang X, Wang S, et al. NOD-Like Receptor Protein 3 InflammasomeDependent IL-1 $\beta$ Accelerated ConA-Induced Hepatitis. Front Immunol. 2018; 9: 758.

49. Czaja AJ, Manns MP. Advances in the diagnosis, pathogenesis, and management of autoimmune hepatitis. Gastroenterology. 2010; 139: 58-72.e4.

50. Longhi MS, Ma Y, Mieli-Vergani G, et al. Aetiopathogenesis of autoimmune hepatitis. J Autoimmun. 2010; 34: 7-14.

51. Bartneck M, Fech V, Ehling J, et al. Histidine-rich glycoprotein promotes macrophage activation and inflammation in chronic liver disease. Hepatology. 2016; 63: 1310-24.

52. Ambade A, Lowe P, Kodys $K$, et al. Pharmacological Inhibition of CCR2/5 Signaling Prevents and Reverses Alcohol-Induced Liver Damage, Steatosis, and Inflammation in Mice. Hepatology. 2019; 69: 1105-21.

53. Rieber N, Gille C, Köstlin N, et al. Neutrophilic myeloid-derived suppressor cells in cord blood modulate innate and adaptive immune responses. Clin Exp Immunol. 2013; 174: 45-52

54. Liu Y, Perego M, Xiao Q, et al. Lactoferrin-induced myeloid-derived suppressor cell therapy attenuates pathologic inflammatory conditions in newborn mice. J Clin Invest. 2019; 129: 4261-75.

55. Bunt SK, Sinha P, Clements VK, et al. Inflammation induces myeloid-derived suppressor cells that facilitate tumor progression. J Immunol. 2006; 176: 284-90.

56. Cripps JG, Wang J, Maria A, et al. Type $1 \mathrm{~T}$ helper cells induce the accumulation of myeloid-derived suppressor cells in the inflamed Tgfb1 knockout mouse liver. Hepatology. 2010; 52: 1350-9.

57. Zhang X, Majlessi L, Deriaud E, et al. Coactivation of Syk kinase and MyD88 adaptor protein pathways by bacteria promotes regulatory properties of neutrophils. Immunity. 2009; 31: 761-71.

58. Li YM, Liu ZY, Wang JC, et al. Receptor-Interacting Protein Kinase 3 Deficiency Recruits Myeloid-Derived Suppressor Cells to Hepatocellular Carcinoma Through the Chemokine (C-X-C Motif) Ligand 1-Chemokine (C-X-C Motif) Receptor 2 Axis. Hepatology. 2019; 70: 1564-81. 